EPJ Web of Conferences 7, 03002 (2010)

DOI:10.1051/epjconf/20100703002

(C) Owned by the authors, published by EDP Sciences, 2010

\title{
Isolated Neutron Stars
}

\author{
Sergei Popov
}

Sternberg Astronomical Institute, Moscow, Russia

\begin{abstract}
Several aspects related to astrophysics of isolated neutron stars are discussed. We start with an introduction into the "new zoo" of young isolated neutron stars. In addition to classical radio pulsars, now we know several species (soft gamma-ray repeators, anomalous X-ray pulsars, central compact objects in supernova remnants, close-by cooling neutron stars - aka "Magnificent seven", - RRATs, and some others). All these types are briefly discussed. In the second lecture a description of magneto-rotational evolution of neutron stars is given. Finally, in the third lecture we discuss population synthesis of isolated neutron stars. In some details we discuss population synthesis of young isolated radio pulsars and young close-by cooling neutron stars.
\end{abstract}

This is an Open Access article distributed under the terms of the Creative Commons Attribution-Noncommercial License 3.0, which permits unrestricted use, distribution, and reproduction in any noncommercial medium, provided the original work is properly cited. 


\title{
Lecture 1 \\ Isolated Neutron Stars. Intro.
}

\author{
Sergei Popov (SAI MSU)
}

Dubna "Dense Matter In Heavy lon Collisions and Astrophysics", July 2008

\section{Artistic view}
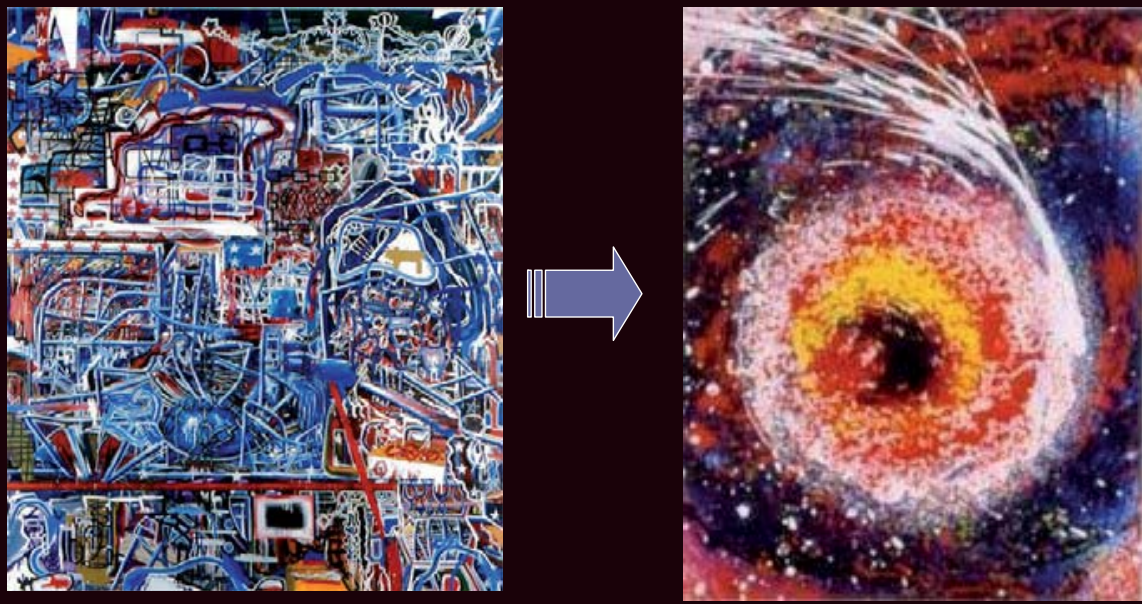


\section{Prediction ...}

Neutron stars have been predicted in 30s:

L.D. Landau: Star-nuclei (1932) + anecdote

Baade and Zwicky:

neutron stars and supernovae (1934)

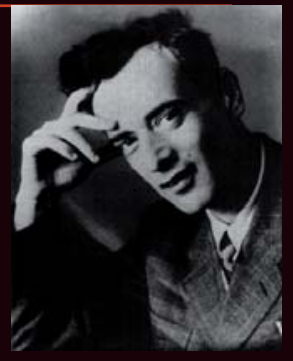

(Landau)
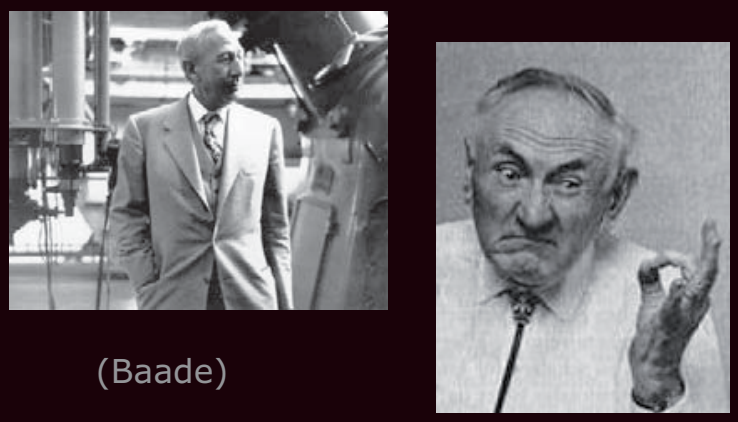

(Zwicky)

(Baade)

Good old classics

For years two main types of NSs have been discussed: radio pulsars and accreting NSS in close binary systems

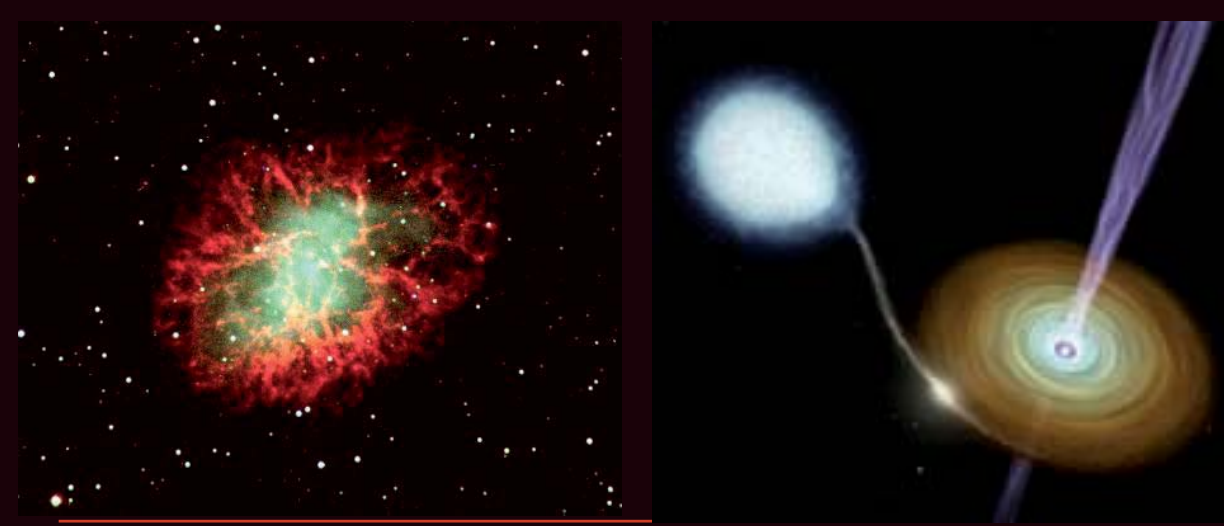

The pulsar in the Crab nebula A binary system 


\section{The old zoo of neutron stars}

In 60s the first X-ray sources have been discovered.

They were neutron stars in close binary systems, BUT ...

.... they were $\ll$ not recognized»....

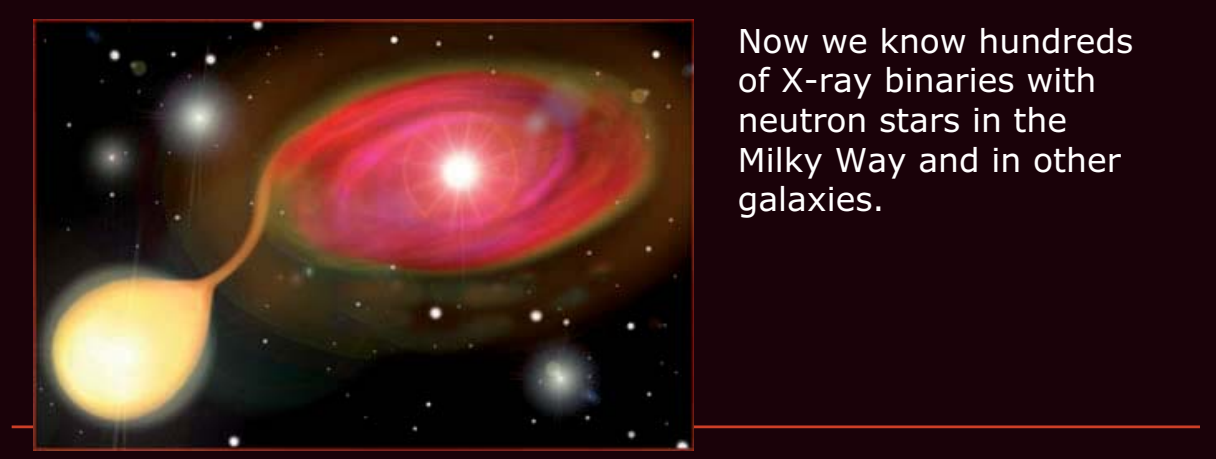

\section{Rocket experiments}

\section{Sco X-1}

Giacconi, Gursky, Hendel 1962

In 2002 R. Giacconi was awarded with the Nobel prize.

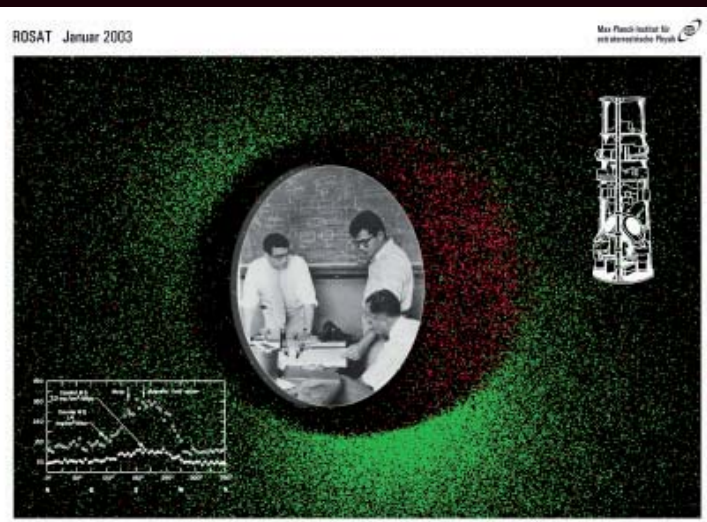




\section{Discovery !!!!}

1967: Jocelyn Bell. Radio pulsars.

Seredipitous discovery.
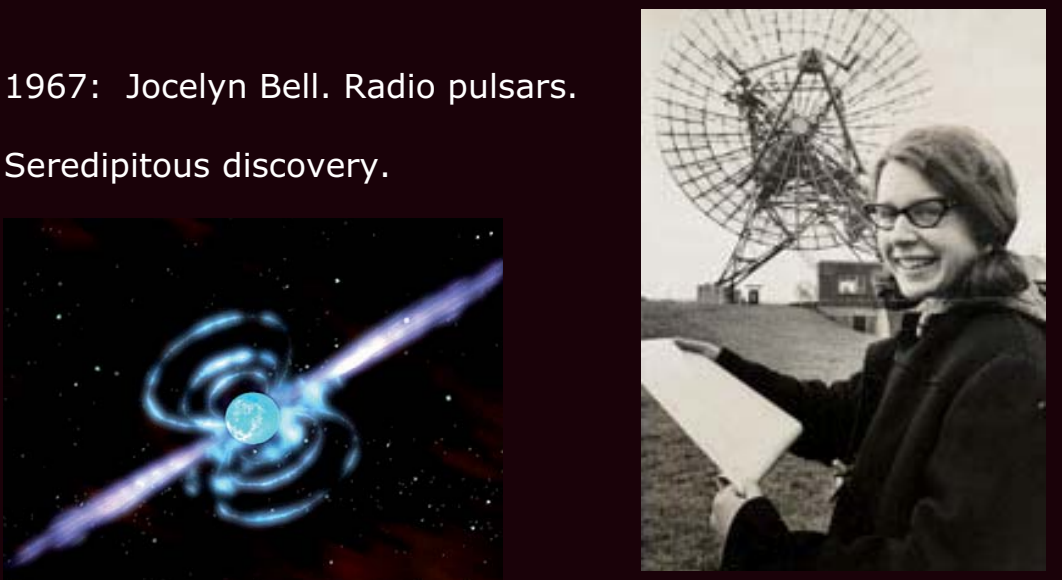

The pulsar in the Crab nebula

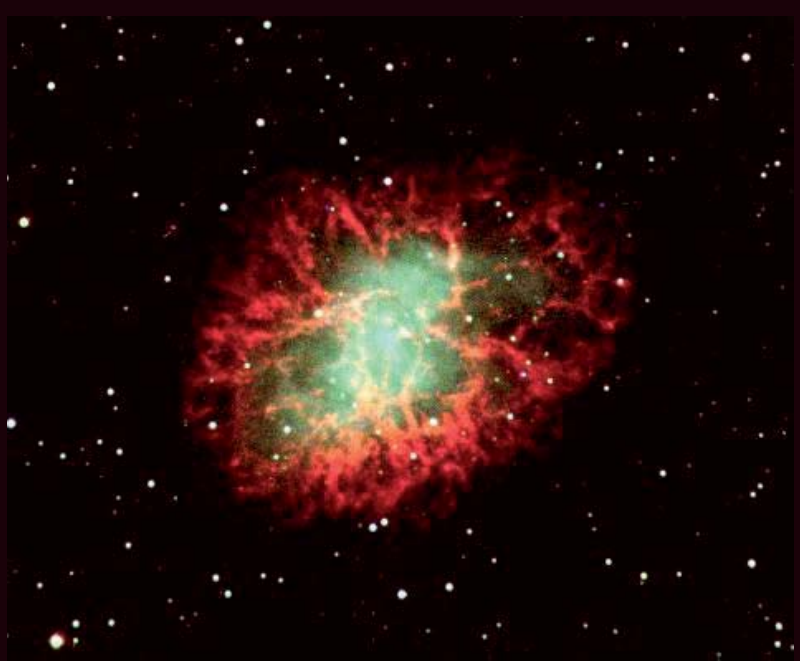




\section{The new zoo of neutron stars}

During last $>10$ years

it became clear that neutron stars can be born very different.

In particular, absolutely non-similar to the Crab pulsar.

- Compact central X-ray sources

in supernova remnants.

- Anomalous X-ray pulsars

o Soft gamma repeaters

o The Magnificent Seven

o Unidentified EGRET sources

o Transient radio sources (RRATs)

o Calvera ....

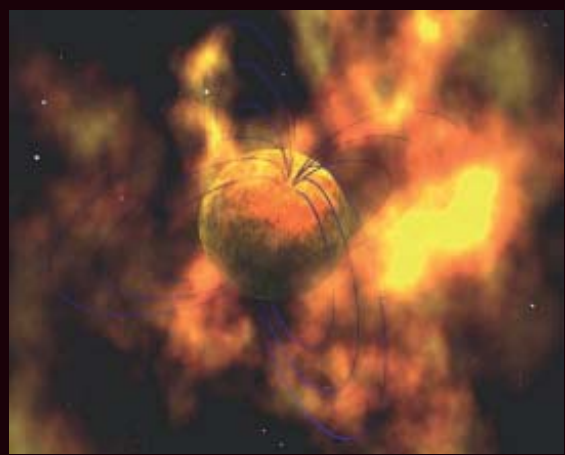

\section{Compact central X-ray sources in}

\section{supernova remnants}

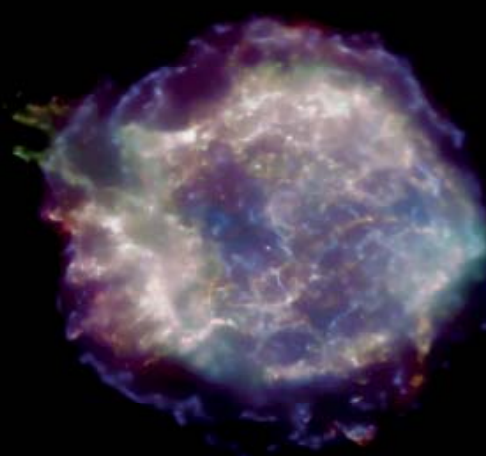

Cas A

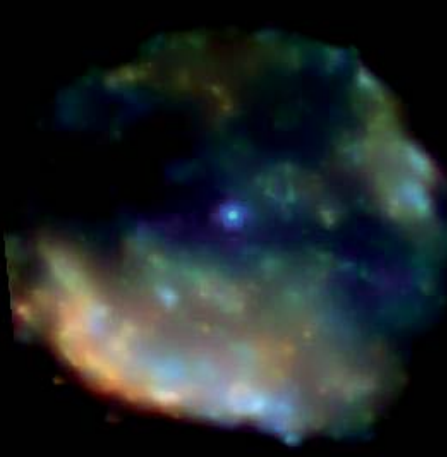

RCW 103 


\section{Puppis A}

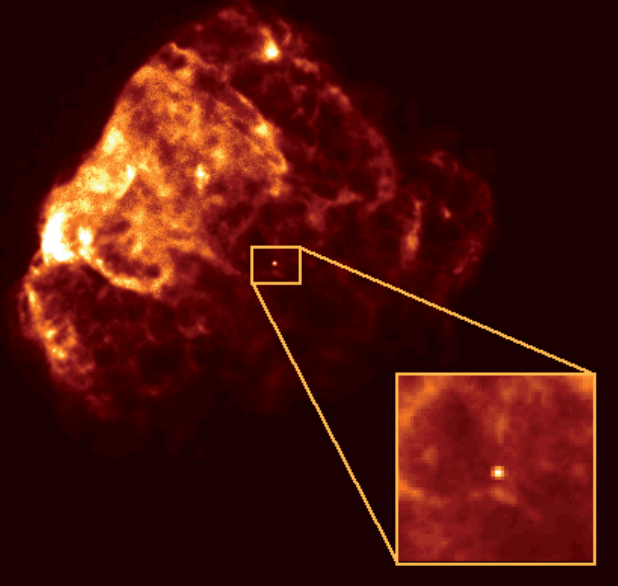

One of the most famous central compact X-ray sources in supernova remnants.

Age about 3700 years.

Probably the progenitor was a very massive star

(mass about 30 solar).

$\mathrm{V}_{\text {kick }}=1500 \mathrm{~km} / \mathrm{s}$ Winkler, Petre 2006 (astro-ph/0608205)

\section{Magnetars}

- $\mathrm{dE} / \mathrm{dt}>\mathrm{dE}_{\mathrm{rot}} / \mathrm{dt}$

- By definition: The energy of the magnetic field is released

- P-Pdot

- Direct measurements of the field (Ibrahim et al.)

$$
\text { Magnetic fields } 10^{14}-10^{15} \mathrm{G}
$$




\section{Known magnetars}

\section{SGRS}

- 0526-66

- 1627-41

- 1806-20

- $1900+14$

- +candidates

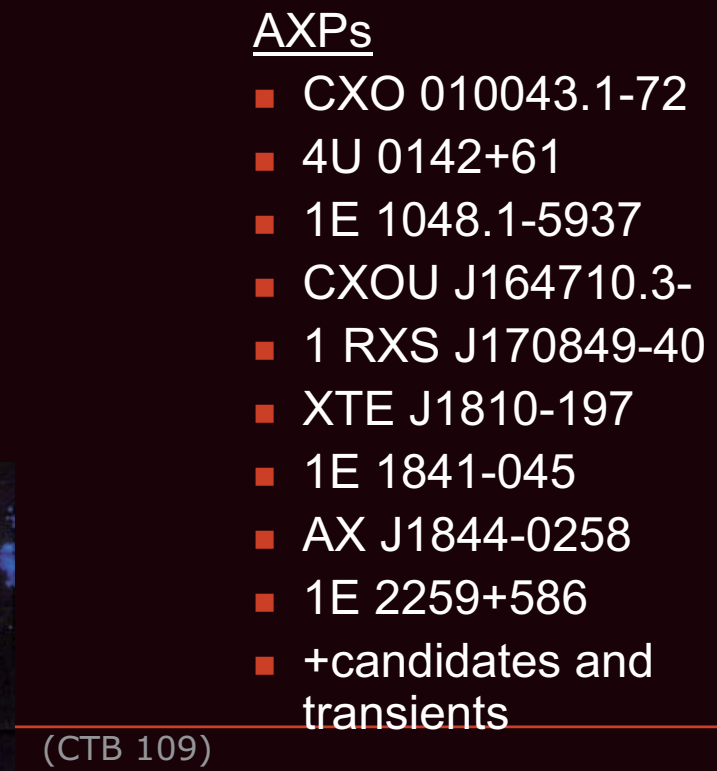

\section{SGRs: monitoring and extraG}

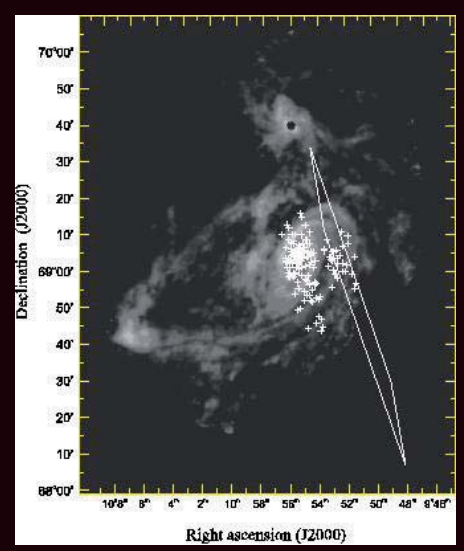

SRG detectors can contribute to observations of SGRs.

Now there are few other candidates

(Mazets et al., Frederiks et al., Golenetskii et al., Ofek et al, Crider ....), including one in the direction of M31 (Mazets et al. arxiv:0712.1502).

[D. Frederiks et al. astro-ph/0609544] 


\section{QPOs after giant flares}

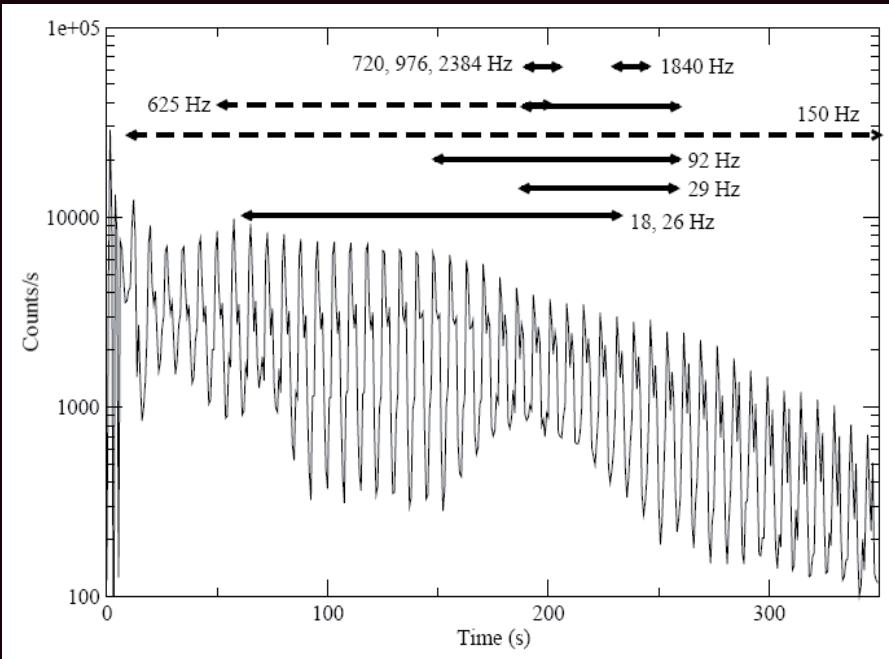

A kind of quasi periodic oscillations have been found in tail of two events (aug. 1998, dec. 2004) They are supposed to be torsional oscillations of NSs, however, it is not clear, yet.

(Israel et al. 2005 astro-ph/0505255,

Watts and Strohmayer 2005 astro-ph/0608463)

See a recent review in aXiv: 0710.2475

\section{Transient radio emission from AXP}

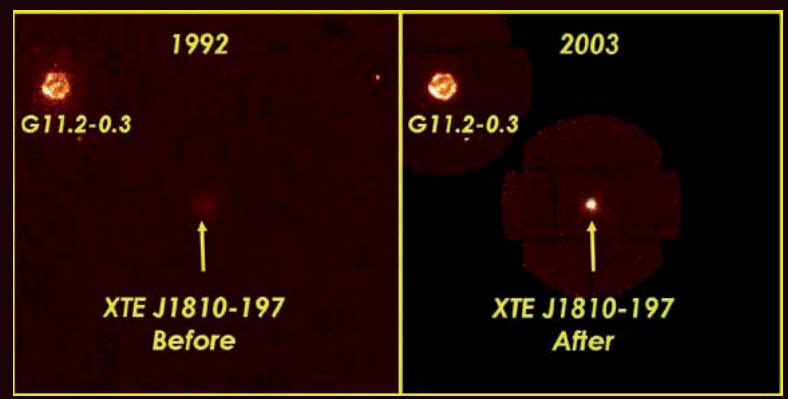

Radio emission was detected from XTE 1810-197 during its active state.

One another magnetar was reported to be detected at low frequencies in Pushchino, however, this result has to be checked.

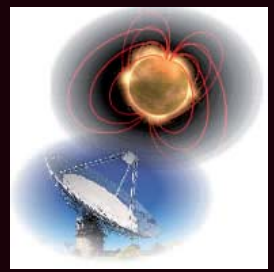




\section{Another AXP detected in radio}

1E 1547.0-5408

$P=2$ sec

SNR G327.24-0.13

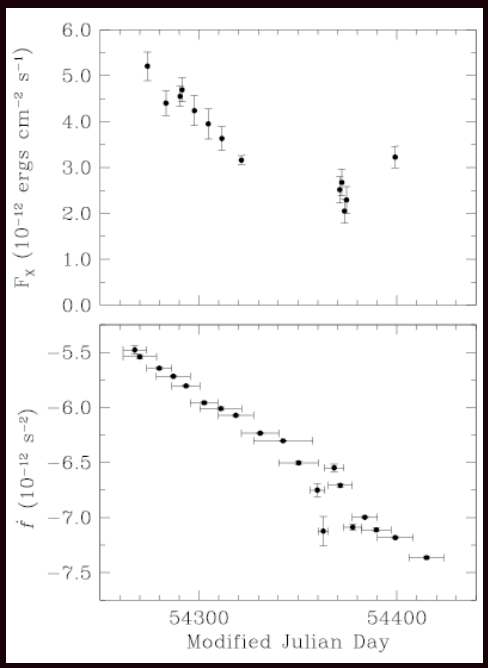

\section{Transient radiopulsar}

\section{PSR J1846-0258}

$P=0.3 \mathrm{sec}$

$B=510^{13} G$

The pulsar increased its luminosity in X-rays.

Magnetar-like X-ray bursts.

\section{Chandra X-ray Image of}

Supernova Remnant Kes 75 and its Central Pulsar

PSR J1846-0258:

The Youngest Known Pulsar, 700 yrs-old

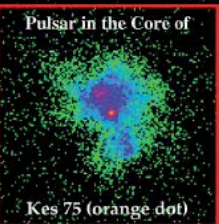

Pulsar

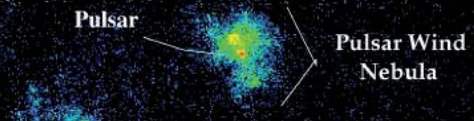

Thermal Shell

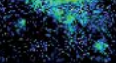

Chandra/ACIS X-ray Snapshot of Oct 102000 


\section{ROSAT}

\section{ROentgen SATellite}

\section{ROSAT}

German satellite

(with participation of US and UK).

Launched 01 June 1990.

The program was successfully ended on 12 Feb 1999.

\section{Close-by radioquiet NSs}

- Discovery: Walter et al. (1996)

- Proper motion and distance: Kaplan et al.

- No pulsations

- Thermal spectrum

- Later on: six brothers

RX J1856.5-3754 


\section{Magnificent Seven}

\begin{tabular}{|l|c|}
\hline Name & Period, s \\
\hline RX 1856 & 7.05 \\
\hline RX 0720 & 8.39 \\
\hline RBS 1223 & 10.31 \\
\hline RBS 1556 & $6.88 ?$ \\
\hline RX 0806 & 11.37 \\
\hline RX 0420 & 3.45 \\
\hline RBS 1774 & 9.44 \\
\hline
\end{tabular}

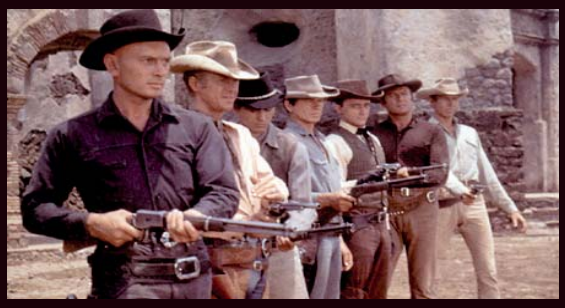

Radioquiet (?) Close-by Thermal emission Absorption features Long periods

\section{Unidentified EGRET sources}

Grenier (2000), Gehrels et al. (2000)

Unidentified sources are divided into several groups.

One of them has sky distribution similar to the Gould Belt objects.

It is suggested that GLAST (and, probably, AGILE)

Can help to solve this problem.

Actively studied subject

(see for example papers by Harding, Gonthier)

No radio pulsars in

56 EGRET error boxes (Crawford et al. 2006)
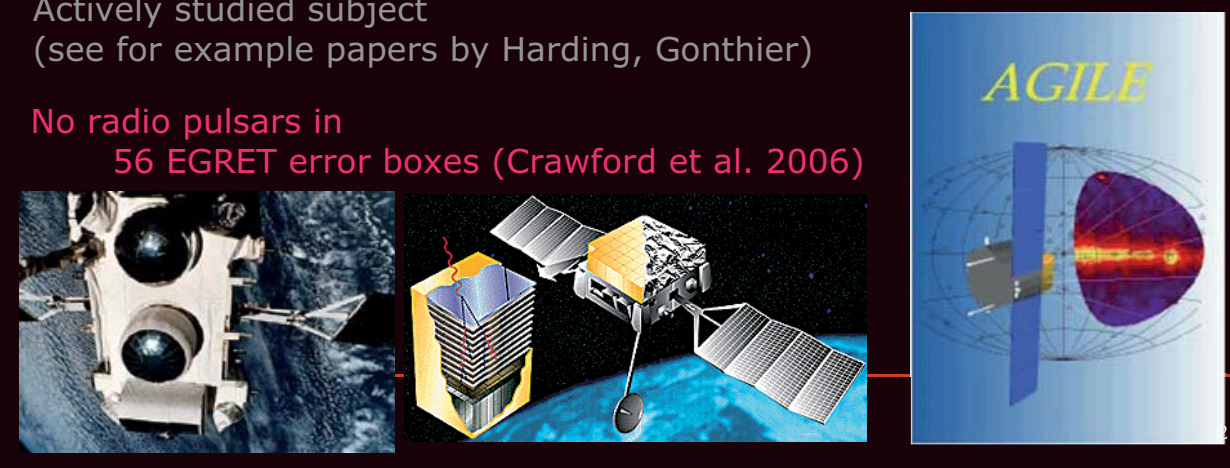


\section{Pulsars invisible in radio?}

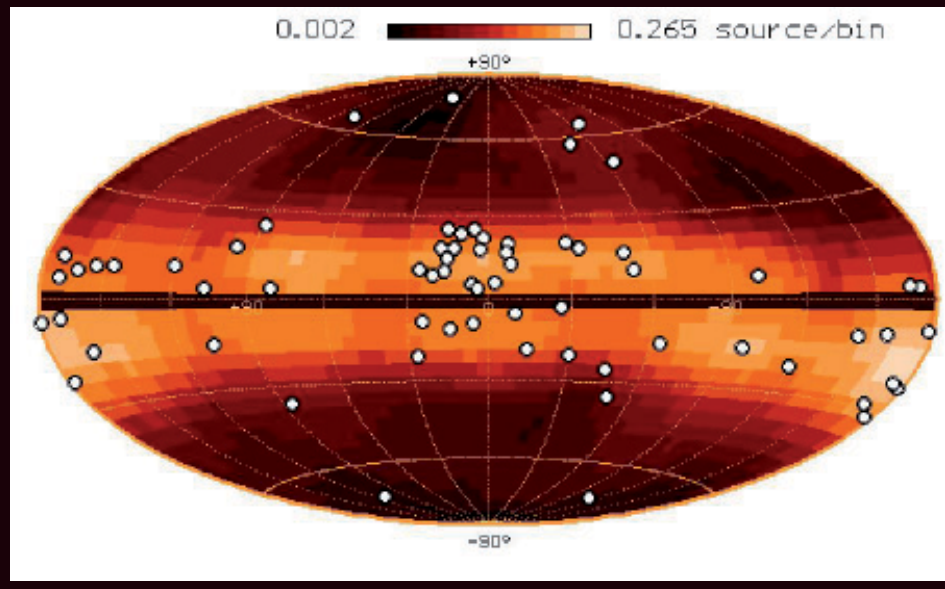

(Grenier astro-ph/0011298)

\section{Discovery of}

radio transients

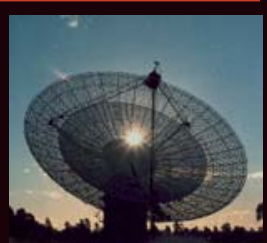

McLaughlin et al. (2006) discovered a new type of sources- RRATs

(Rotating Radio Transients).

For most of the sources periods about few seconds were discovered. The result was obtained during the Parkes survey of the Galactic plane.

These sources can be related to The Magnificent seven.

Thermal X-rays were observed from one of the RRATs (Reynolds et al. 2006). This one seems to me the youngest. 


\section{P-Pdot diagram for RRATs}

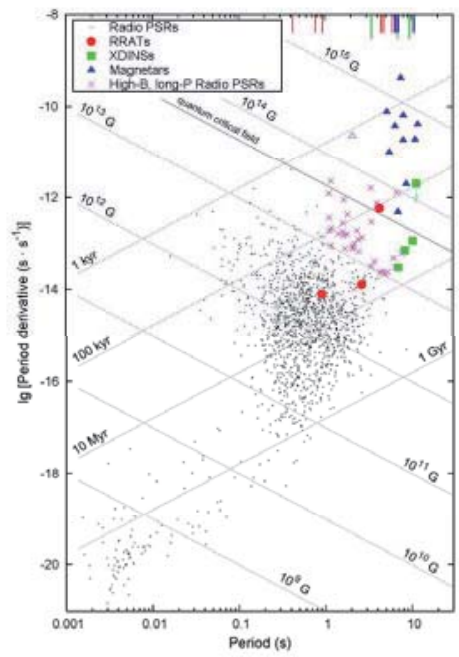

McLaughlin et al. 2006 Nature

Estimates show that there should be about 400000

Sources of this type in the Galaxy.

Young or old???

Relatives of the Magnificent seven? (astro-ph/0603258)

\section{RRATs}

- 11 sources detected in the Parkes Multibeam survey (McLaughlin et al 2006)

- Burst duration 2-30 ms, interval 4 min-3 hr

- Periods in the range 0.4-7 s

- Period derivative measured in 3 sources: B $10^{12}-10^{14} \mathrm{G}$, age $\sim 0.1-3 \mathrm{Myr}$

- RRAT J1819-1458 detected in the X-rays, spectrum soft and thermal, kT $\sim 120 \mathrm{eV}$ (Reynolds et al 2006) 


\section{RRATs}

- P, B, ages and X-ray properties of RRATs very similar to those of XDINSs

- Estimated number of RRATs $\sim 3-5$ times that of PSRs

口 If $\mathrm{T}_{\mathrm{RRAT}} \approx \mathrm{T}_{\mathrm{PSR}}, \beta_{\mathrm{RRAT}} \approx 3-5 \beta_{\mathrm{PSR}}$

- $\beta_{\text {XDINS }}>3 \beta_{\text {PSR }}$ (Popov et al 2006)

- Are RRATs far away XDINSs ?

\section{RRATs. Recent data}

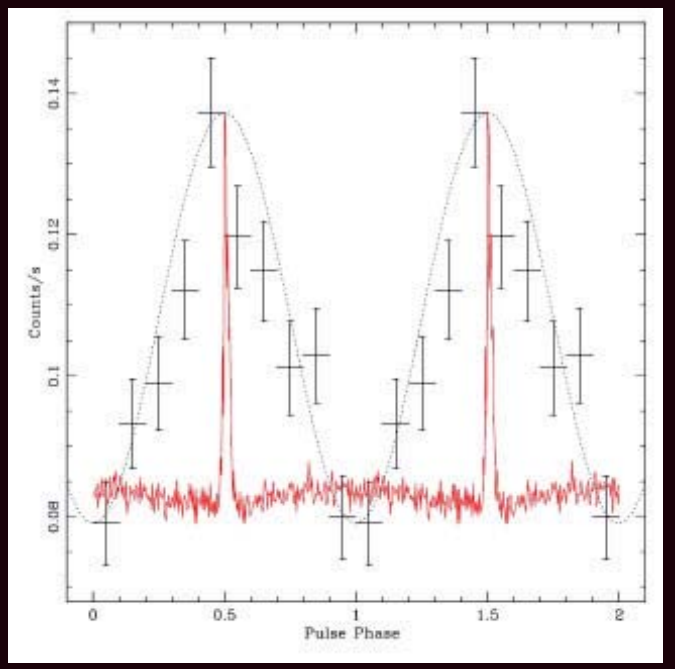

X-ray pulses overlaped on radio data of RRAT J1819-1458. 


\section{Calvera et al.}

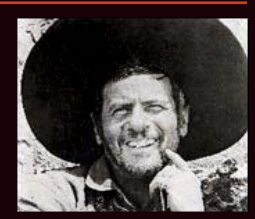

Recently, Rutledge et al. reported the discovery of an enigmatic NS candidated dubbed Calvera.

It can be an evolved (aged) version of Cas A source, but also it can be a M7-like object, who's progenitor was a runaway (or, less probably, hypervelocity) star.

No radio emission was found (arxiv:0710.1788).

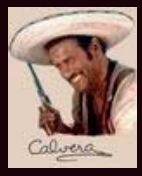

\section{LOFAR}

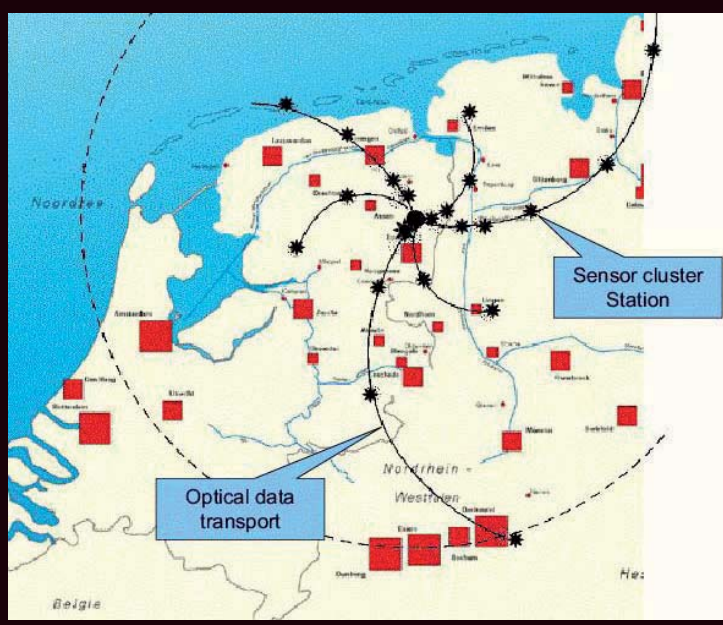

Low Frequency Array (<250 MHz) Perfect for wide-field observations search for transients in radio etc. 


\section{Gravitational waves from INSs}

INS are expected to be sources of GWs:

- Radio pulsars

- Young magnetars

-.......?

- Know periods

- Rapid rotation and strong deformation

- Possible new types sources

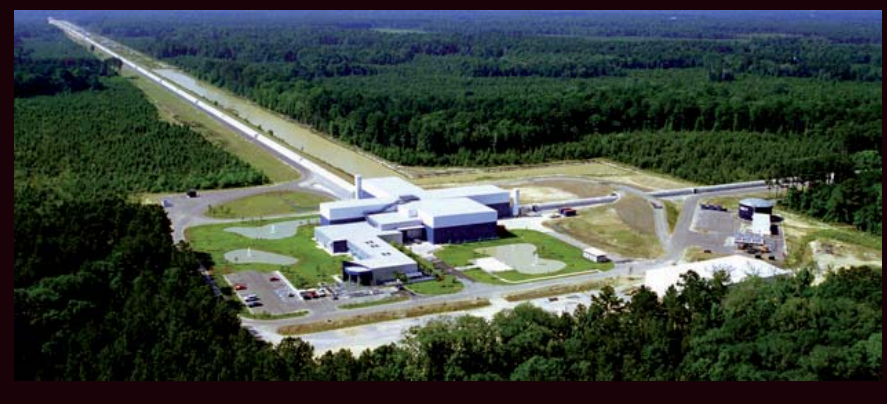

\section{Recent LIGO results for PSRs}

1. 0805.4758 Beating the spin-down limit on gravitational wave emission from the Crab pulsar

$\mathrm{h}_{0} 95 \%<3.510^{-25} \varepsilon<1.910^{-4}$ (single template)

2. 0708.3818 All-sky search for periodic grav. waves in LIGO S4 data

\section{0-1000 HZ}

No evidence. Upper limits on isolated NSs GW emission. Very weak limits for "dark" sources ( $<50$ pc for $\varepsilon \sim 10^{-6}$ )

3. gr-qc/0702039 Upper limits on gravitational wave emission from 78 PSRs $\varepsilon<10^{-6}$ for PSR J2124-3358

$\mathrm{h}<2.6 \times 10^{-25}$ for PSR J1603-7202 


\section{GWs from young magnetars}

A newborn magnetar with msec period and toroidal magnetic field $B \sim 10^{16} \mathrm{G}$ for few days can be a strong source detectable from the Virgo cluster distance by advanced LIGO.

Birthrate of magnetars in Virgo is about $0.3-1$ per year.

Due to strong toroidal magnetic fields young magnetars can have prolate shape with ellipticity $10^{-3}-10^{-4}$.

Power of the signal goes as $\sim P^{-6}$.

$$
\dot{\omega}=-K_{d} \omega^{3}-K_{\mathrm{gw}} \omega^{5}
$$

$\tau_{s d} \equiv \frac{\omega}{2 \dot{\omega}} \simeq 10 P_{i, 2}^{2}\left(B_{d, 14}^{2}+1.15 B_{t, 16.3}^{4} P_{i, 2}^{-2}\right)^{-1} \mathrm{~d}$

$h \sim 3 \times 10^{-26} d_{20}^{-1} P_{2}^{-2} B_{t, 16.3}^{2}$

Instantaneous signal

astro-ph/0702075 Dall'Osso et al. astro-ph/0511068 Stella et al.

\section{Detectability by advanced LIGO}

$h_{c}=h N^{1 / 2}$, where $N \simeq \tau_{s d} / P_{i} \quad$ The signal is observable for several periods

$$
h_{c} \simeq 6 \times 10^{-22} \frac{B_{t, 16.3}^{2}}{d_{20} P_{i, 2}^{3 / 2}\left(B_{d, 14}^{2}+1.15 B_{t, 16.3}^{4} P_{i, 2}^{-2}\right)^{\frac{1}{2}}}
$$

So, the signal lasts for several days after a magnetar's birth

It is possible to get very optimistic predictions for advanced LIGO. However, mining the data for a signal with uknown parameters from unknown place in the sky is very complicated.

Computational costs are very large.

astro-ph/0702075 Dall'Osso et al.

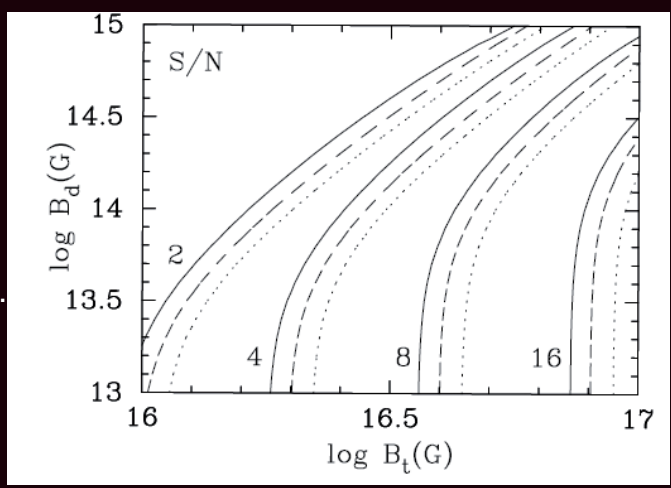




\section{Magnetar parameters}

Estimating GW emission form magnetars it is necessary to make assumptions avout ellipticity. It is not that easy.

\subsection{Pons et al.}

\section{Relativistic models of magnetars: structure and deformations}

This authors estimate that for $B \sim 10^{15} \mathrm{G}$ ellipticity is expected $\sim 10^{-6}-10^{-5}$ In extreme cases (low-mass NSs) it can go up to $10^{-3}$

Crustal field are very important.

\subsection{Regimbau, Mandic}

\section{Astrophysical sources of stochastic GW background}

Magnetars produce strong (in comparison with expected cosmological signal) background around $1 \mathrm{kHz}$ (depends on initil spin of magnetars).

Next generation of interferometers will give important results on NSs.

\section{Population synthesis of close-by NS and GWs}

\section{Palomba [astro-ph/0503046]}

The author assumes that some fraction of NSs are born with low magnetic fields, so that GW losses dominate in spin-down.

The author predicts that few NSs can be detected by Virgo (for not too small fraction of low-field NSs), and tens - by advanced Virgo.

Expected distances (for Virgo) are $100-300$ pc, and frequences about $200-600 \mathrm{~Hz}$. 


\section{Conclusion}

- There are several types of sources: CCOs, M7, SGRs, AXPs, RRATs ...

- Magnetars (?)

- Significant fraction of all newborn NSs

- Unsolved problems:

1. Are there links?

2. Reasons for diversity
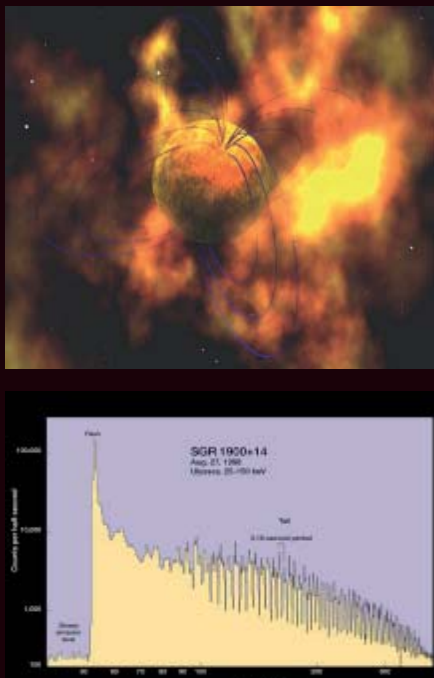

ras

\section{Main reviews}

-NS basics:

-SGRs \& AXPs:

-CCOs:

-Quark stars:

-The Magnificent Seven:

-RRATs:

-Cooling of NSs:

- NS structure

- NS interiors

- Magnetic field of NSs

- EoS

- NS atmospheres physics/0503245

astro-ph/0405262

astro-ph/0406133

astro-ph/0311526

0712.2209

astro-ph/0608360

astro-ph/0502457

astro-ph/0609066

0801.1143

astro-ph/0511587

astro-ph/0508056

astro-ph/0402143

astro-ph/0608360

arXiv: 0705.2708

arXiv: 0802.2227

0711.3650

astro-ph/0612440

astro-ph/0206025 


\title{
Lecture 2 Spin evolution of NSs
}

\author{
Sergei Popov (SAI MSU)
}

Dubna "Dense Matter In Heavy lon Collisions and Astrophysics", July 2008

\section{Hard life of neutron stars}

There are about $610^{9}$ persons on Earth. How many do you know?

There are about $10^{9}$ NSs in the Galaxy. How many do we know? Why?

We know PSRs, SGRs, AXPs, CCOs, M7, RRATs, .... They are young.

\section{Dialogue of two magnetars:}

-We are not getting younger, man....

-Yeh, at first you lose spin, then - magnetic field, and then you just cool down... - ... and nobody cares about you any more ....

\section{Evolution is important!!!}




\section{Evolution of neutron stars}

\section{Thermal}

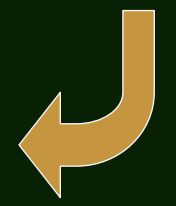

\section{Magneto- rotational}

Observational appearence of a NS can depend on:

- Temperature

- Period

- Magnetic field

- Velocity

\section{Evolution of NSs: temperature}

Neutrino

cooling stage

\section{Photon}

cooling stage

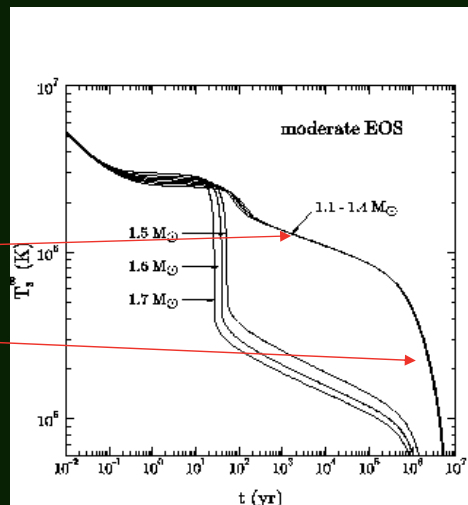

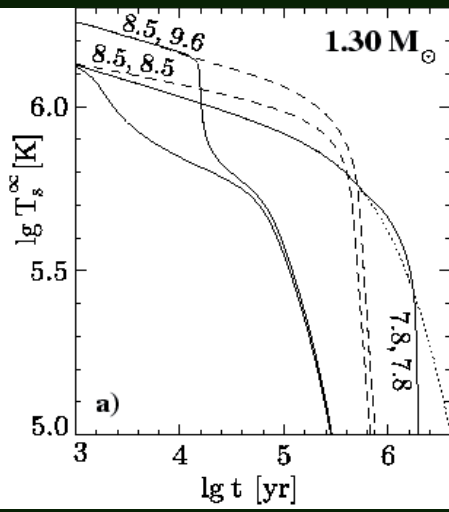

[Yakovlev et al. (1999) Physics Uspekhi]

First papers on the therma

evolution appeared already

in early 60 s, i.e. before

the discovery of radio pulsars. 


\section{Evolution of neutron stars:}

\section{rotation + magnetic field}

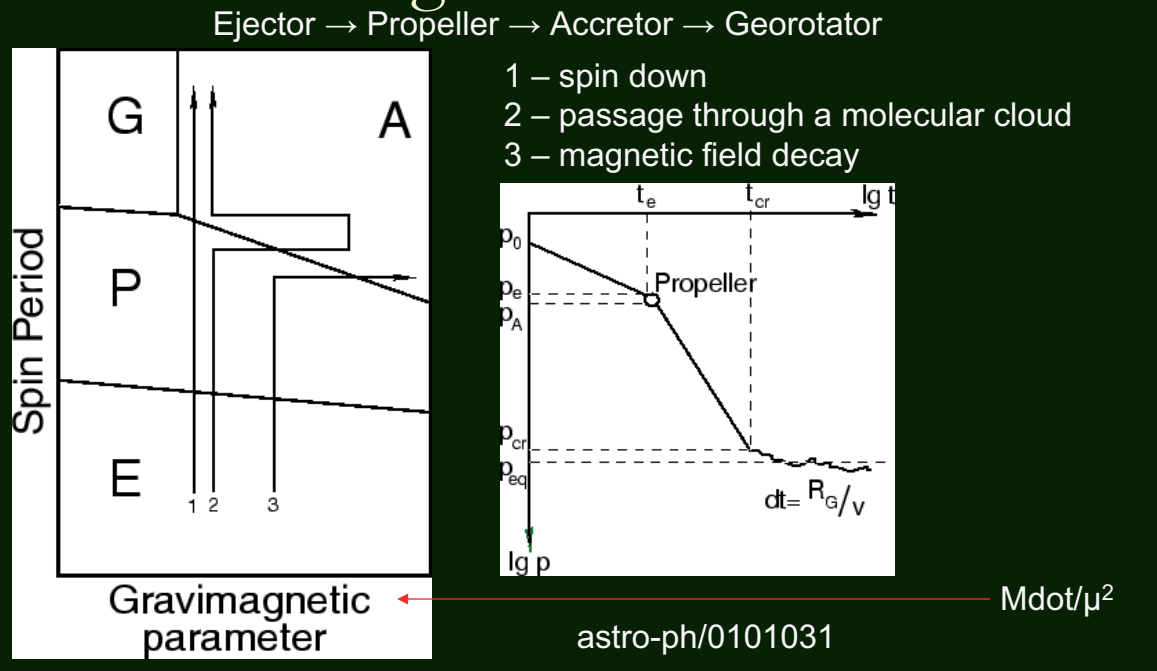

See the book by Lipunov $(1987,1992)$

\section{Magnetic rotator}

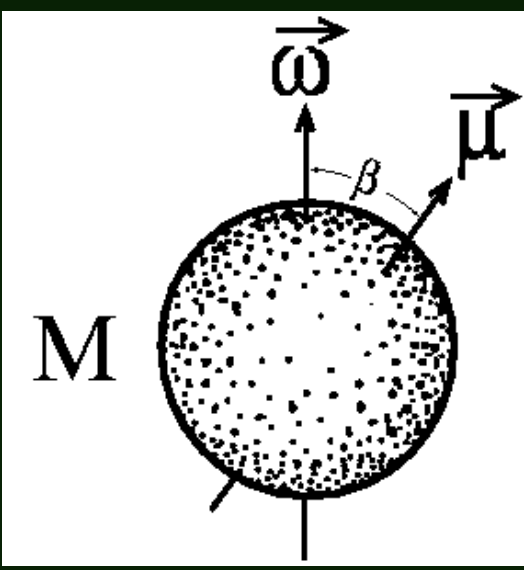

Observational appearences of NSs

(if we are not speaking about cooling)

are mainly determined by $\mathrm{P}, \mathrm{Pdot}, \mathrm{V}, \mathrm{B}$,

(probably the inclination angle $\mathrm{X}$ ),

and properties of the surrounding medium.

$B$ is not evolving significantly in most cases,

so it is important to discuss spin evolution.

\section{Together with changes in B (and X) one can speak about \\ magneto-rotational evolution}

We are going to discuss the main stages of this evolution, namely:

Ejector, Propeller, Accretor, and Georotator following the classification by Lipunov 


\section{Magneto-rotational evolution of radio pulsars}

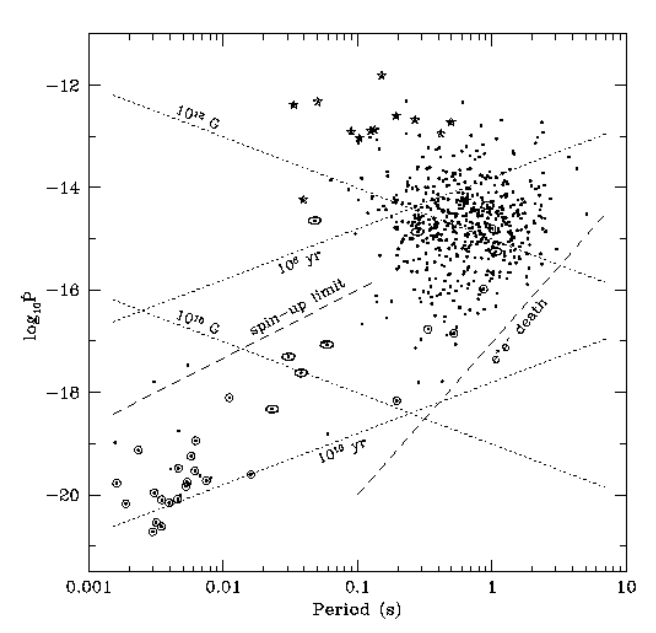

For radio pulsar magneto-rotational evolution is usually illustrated in the P-Pdot diagram.

However, we are interested also in the evolution after this stage.

$L_{m}=\frac{2}{3} \frac{\mu^{2} \omega^{4}}{\omega^{3}} \sin ^{2} \beta=\kappa_{t} \frac{\mu^{2}}{R_{l}^{3}} \omega$

$B \sim 3.2 \times 10^{19}(P d P / d t)^{1 / 2} \mathrm{G}$.

Spin-down.

Rotational energy is released.

The exact mechanism is still unknown.

\section{Magneto-rotational evolution of NSs}

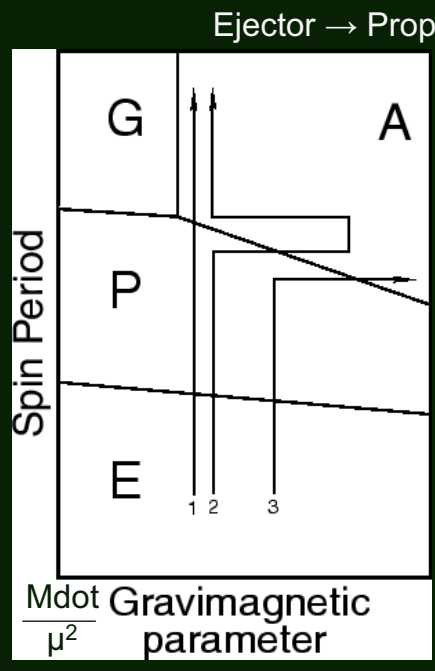

$$
\begin{aligned}
& 1 \text { - spin down } \\
& 2 \text { - passage through a molecular cloud } \\
& 3 \text { - magnetic field decay }
\end{aligned}
$$

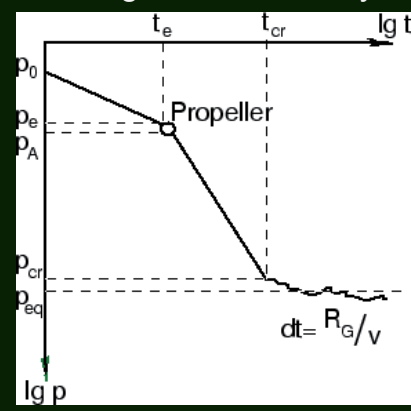

astro-ph/0101031

See the book by Lipunov $(1987,1992)$ 


\section{Critical radii -I}

Transitions between different evolutionary stages can be treated in terms of critical radii

- Ejector stage. Radius of the light cylinder. $R_{l}=c / \omega$.

Shvartsman radius. $\mathrm{R}_{\mathrm{sh}}$.

- Propeller stage. Corotation radius. $\mathrm{R}_{\mathrm{co}}$

- Accretor stage. Magnetospheric (Alfven) radius. $R_{A}$

- Georotator stage. Magnetospheric (Alfven) radius. $\mathrm{R}_{\mathrm{A}}$

As observational appearence is related to interaction with the surrounding medium the radius of gravitational capture is always important. $R_{G}=2 G M / N^{2}$.

Schwarzshild radii is typicall unimportant.

$$
r_{g}=\frac{2 G M}{c^{2}} \approx 2.95 \frac{M}{M_{S U N}} \mathrm{~km}
$$

\section{Critical radii-II}

1. Shvartsman radius

It is determined by

relativistic particles wind

$$
R_{\mathrm{Sh}}=\left(\frac{8 \kappa_{t} \mu^{2} G^{2} M^{2} \dot{\omega}^{4}}{\dot{M}_{c} v_{\infty}^{5} c^{4}}\right)^{1 / 2}, \quad R_{\mathrm{Sh}}>R_{G}
$$

2. Corotation radius

$$
\omega R_{\mathrm{St}}<\sqrt{G M_{x} / R_{\mathrm{St}}}
$$

$$
R_{c}=\left(G M M_{x} / \omega^{2}\right)^{1 / 3} \sim 2.8 \times 10^{8} m_{x}^{1 / 3}(P / 1 \mathrm{~s})^{2 / 3} \mathrm{~cm}
$$

3. Alfven radius

$$
P_{m}\left(R_{\mathrm{st}}\right)=P_{a}\left(R_{\mathrm{st}}\right)
$$

$R_{\Lambda}= \begin{cases}\left(\frac{2 \mu^{2} G^{2} M^{2}}{\dot{M}_{c} v_{\infty}^{5}}\right)^{1 / 6}, & R_{\Lambda}>R_{G} \\ \left(\frac{\mu^{2}}{2 \dot{M}_{c} \sqrt{2 G M}}\right)^{2 / 7}, & R_{\Lambda} \leq R_{G}\end{cases}$ 


\section{Pressure}

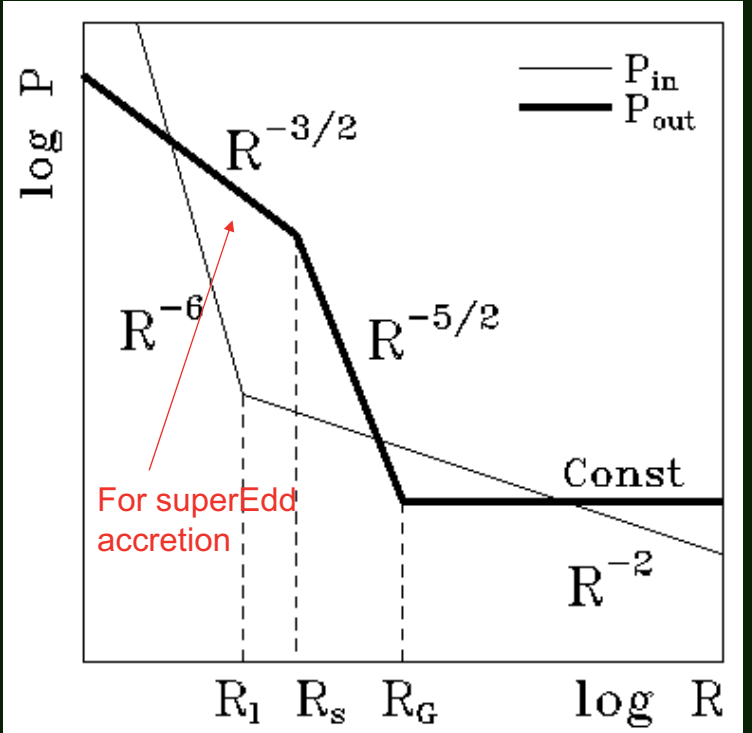

$P_{m}=\left\{\begin{array}{cl}\frac{\mu^{2}}{8 \pi R^{6}}, & R \leq R_{i} \\ \frac{L_{m}}{4 \pi R^{2} c}, & R>R_{i}\end{array}\right.$

$L_{m}=\kappa_{t} \frac{\mu^{2}}{R_{i}^{3}} \omega$

We can define a stopping radius $\mathrm{R}_{\mathrm{st}}$, at which external and internal pressures are equal.

The stage is determined by relation of this radius to other critial radii.

\section{Classification}

\begin{tabular}{|c|c|c|c|c|}
\hline $\begin{array}{l}\text { Abbrcvi- } \\
\text { ation }\end{array}$ & Турс & $\begin{array}{l}\text { Charactcristic } \\
\text { radii rclation }\end{array}$ & $\begin{array}{l}\text { Accretion } \\
\text { ratc }\end{array}$ & $\begin{array}{l}\text { Obscrvational } \\
\text { appcarances }\end{array}$ \\
\hline $\mathrm{E}$ & Ejcctor & $\begin{array}{l}R_{\mathrm{st}}>R_{G} \\
R_{\mathrm{st}}>R_{t}\end{array}$ & $\dot{M}_{c} \leq \dot{M}_{\mathrm{cr}}$ & $\begin{array}{l}\text { Radiopulsars, } \\
\text { Soft } \gamma \text {-ray } \\
\text { repcatcrs, } \\
\text { Cyg X-3? } \\
\text { LSI+61 } 303 ?\end{array}$ \\
\hline $\mathrm{P}$ & Propeller & $\begin{array}{l}R_{c}<R_{\mathrm{st}} \\
R_{\mathrm{st}} \leqq R_{G} \\
R_{\mathrm{st}} \leq R_{\mathrm{t}}\end{array}$ & $\dot{M}_{c} \leq \dot{M}_{\mathrm{cr}}$ & $\begin{array}{l}\text { X-ray transicnts? } \\
\text { Rapid burstcr? } \\
\text { Y-burstcrs??? } \\
\text { Magnctic } \\
\text { Ap-stars }\end{array}$ \\
\hline A & Accretor & $\begin{array}{l}R_{\mathrm{st}} \leq R_{G} \\
R_{\mathrm{st}} \leq R_{t}\end{array}$ & $\dot{M}_{c} \leq \dot{M}_{\mathrm{cr}}$ & $\begin{array}{l}\text { X-ray pulsars, } \\
\text { burstcrs, } \\
\text { cataclysmic } \\
\text { variables, } \\
\text { intermediatc } \\
\text { polars }\end{array}$ \\
\hline G & Gcorotator & $\begin{array}{ll}\mathrm{r} & R_{G}<R_{\mathrm{st}} \\
& R_{\mathrm{st}} \leq R_{c}\end{array}$ & $\dot{M}_{c} \leq \dot{M}_{\mathrm{cr}}$ & $\begin{array}{l}\text { Earth, } \\
\text { Jupiter }\end{array}$ \\
\hline M & Magnetor & $\begin{array}{l}R_{\mathrm{st}}>a \\
R_{c}>a ? ? ?\end{array}$ & $\dot{M}_{c} \leq \dot{M}_{\mathrm{cr}}$ & $\begin{array}{l}\text { AM Hor, } \\
\text { polars }\end{array}$ \\
\hline
\end{tabular}




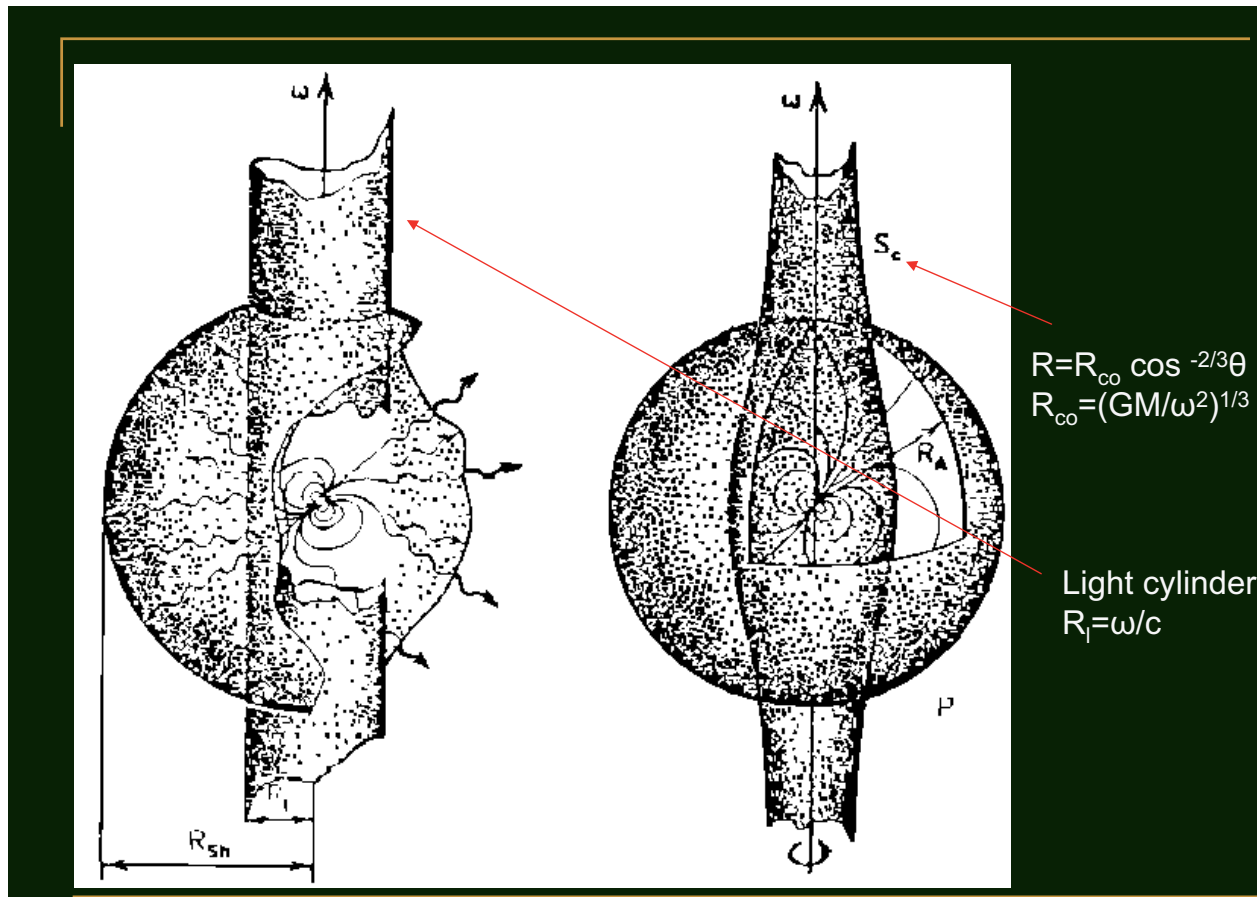

Ejector

Propeller

13

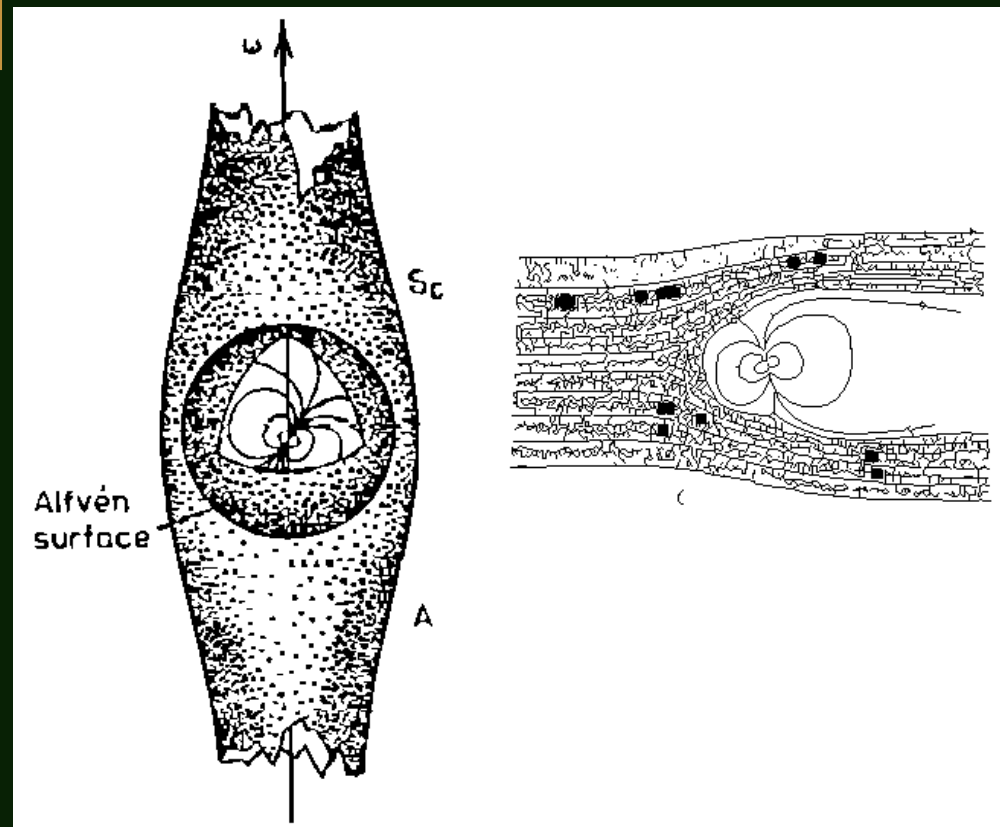




\section{Unified approach to spin-down}

One can find it comfortable to represent the spin-down moment by such a formula

$$
-\kappa_{t} \frac{\mu^{2}}{R_{t}^{3}}
$$

$k_{t}$ and $R_{t}$ are different for different stages.

$k_{t}$ can be also frequency dependent.

\begin{tabular}{lcccccc} 
Paramctcr & \multicolumn{6}{c}{ Regimc } \\
\cline { 2 - 7 } & E, SE & P, SP & A & SA & G & M \\
\hline$\dot{M}$ & 0 & 0 & $\dot{M}_{c}$ & $\dot{M}_{c}\left(R_{A} / R_{s}\right)$ & 0 & $\dot{M}_{c}$ \\
$\kappa_{t}$ & $\sim 2 / 3$ & $\lesssim 1 / 3$ & $\sim 1 / 3$ & $\sim 1 / 3$ & $\sim 1 / 3$ & $\sim 1 / 3$ \\
$R_{t}$ & $R_{t}$ & $R_{m}$ & $R_{c}$ & $R_{c}$ & $R_{\Lambda}$ & $a$
\end{tabular}

\section{Spin-up/down at the stage of accretion}

$\frac{d I \omega}{d t}=\dot{M} k_{\mathrm{su}}-\kappa_{t} \frac{\mu^{2}}{R_{t}^{3}}$

$k_{\mathrm{su}}= \begin{cases}\left(G M_{x} R_{d}\right)^{1 / 2}, & \text { Keplcrian disk accrction } \\ \eta_{t} \Omega R_{G}^{2}, & \text { wind accretion in a binary } \\ \sim 0, & \text { a singlo magnetic rotator. }\end{cases}$

For a single rotator (i.e. an isolated NS) spin-up can be possible due to turbulence in the interstellar medium.

In the case of

isolated accreting NS

one can estimate

the accretion rate as:

$$
\dot{M}_{c}=4 \pi R_{G}^{2} \rho_{\infty} v_{\infty}
$$




\section{Equilibrium period}

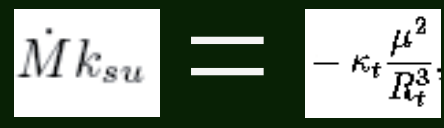

The hypothesis of equilibrium can be used to determine properties of a NS.

The corotation radius is decreasing as a NS is spinning up.

So, before equilibrium is reached the transition to the propeller stage can happen.

Looking at this formula (and remembering that for Accretors $R_{t}=R_{c 0}$ ) it is easy to understand why millisecond PSRs have small magnetic field.

Spin-up can not be very large (Eddington rate).

So, to have small spin periods (and so small corotation radii),

it is necessary to have small magnetic fields.

High magnetic field NS can not be spun-up to millisecond periods.

\section{Critical periods for isolated NSs}

$P_{E}(E \rightarrow P) \simeq 10 \mu_{30}^{1 / 2} n^{-1 / 4} v_{10}^{1 / 2} \mathrm{~s}$

$$
t_{E} \simeq 10^{9} \mu_{30}^{-1} n^{-1 / 2} v_{10} \mathrm{yr}
$$

Transition from Ejector to Propeller (supersonic) Duration of the ejector stage $P_{A}(P \rightarrow A) \simeq 420 \mu_{30}^{6 / 7} n^{-3 / 7} v_{10}^{9 / 7} \mathrm{~s}$

Transition from supersonic Propeller to subsonic Propeller or Accretor

$P_{e q}=2.6 \times 10^{3} v_{(t) 10}^{-2 / 3} \mu_{30}^{2 / 3} n^{-2 / 3} v_{10}^{13 / 3} \mathrm{~s}$

A kind of equilibrium period for the case of accretion from turbulent medium

$v<410 n^{1 / 10} \mu_{30}^{-1 / 5} \mathrm{kms}^{-1}$

Condition for the Georotator formation (instead of Propeller or Accretor)

(see, for example, astro-ph/9910114) 


\section{Accreting isolated neutron stars}

\section{Why are they so important?}

- Can show us how old NSs look like

1. Magnetic field decay

2. Spin evolution

- $\quad$ Physics of accretion at low rates

- NS velocity distribution

- New probe of NS surface and interiors

- ISM probe

\section{Expected properties}

\section{Accretion rate}

An upper limit can be given by the Bondi formula:

Mdot $=\pi R_{G}{ }^{2} \rho v, R_{G} \sim v^{-2}$

Mdot $=10^{11} \mathrm{~g} / \mathrm{s} \quad(\mathrm{v} / 10 \mathrm{~km} / \mathrm{s})^{-3} \mathrm{n}$

$L=0.1$ Mdot $c^{2} \sim 10^{31} \mathrm{erg} / \mathrm{s}$

However, accretion can be smaller due to the influence of a magnetosphere of a NS

\section{Periods}

Periods of old accreting NSs are uncertain, because we do not know evolution well enough.

a)

$p_{\mathrm{A}}=2^{5 / 14} \pi(G M)^{-5 / 7}\left(\mu^{2} / \dot{M}\right)^{3 / 7} \simeq$
$\mathrm{R}_{\mathrm{A}}=\mathrm{R}_{\mathrm{co}}$
$300 \mu_{30}^{6 / 7}\left(v / 10 \mathrm{~km} \mathrm{~s}^{-1}\right)^{9 / 7} n^{-3 / 7} \mathrm{~s}$




\section{Subsonic propeller}

Even after $R_{c o}>R_{A}$ accretion can be inhibited.

This have been noted already in the pioneer papers by Davies et al.

Due to rapid (however, subsonic) rotation a hot envelope is formed around the magnetosphere. So, a new critical period appear.

$$
P_{\mathrm{br}} \simeq 450 \mu_{30}^{16 / 21} \dot{M}_{15}^{-5 / 7} m^{-4 / 21} \mathrm{~s}
$$

(Ikhsanov astro-ph/0310076)

If this stage is realized (inefficient cooling) then

- accretion starts later

- accretors have longer periods

\section{Expected properties-2}

\section{Temperatures}

Depend on the magnetic field. The size of polar caps depends on the field and accretion rate: $\sim R\left(R / R_{A}\right)^{1 / 2}$

\section{Magnetic fields}

Very uncertain, as models of the field decay cannot give any solid predictions

\section{Flux varilability.}

Due to fluctuations of matter density and turbulent velocity in the ISM

it is expected that isolated accretors are variable on a time scale

$\sim \mathrm{R}_{\mathrm{G}} / \mathrm{v} \sim$ days - months

Still, isolated accretors are expected to be numerous at low fluxes (their total number in the Galaxy is large than the number of coolers of comparable luminosity). They should be hotter than coolers, and have much longer spin periods. 


\section{Properties of accretors}
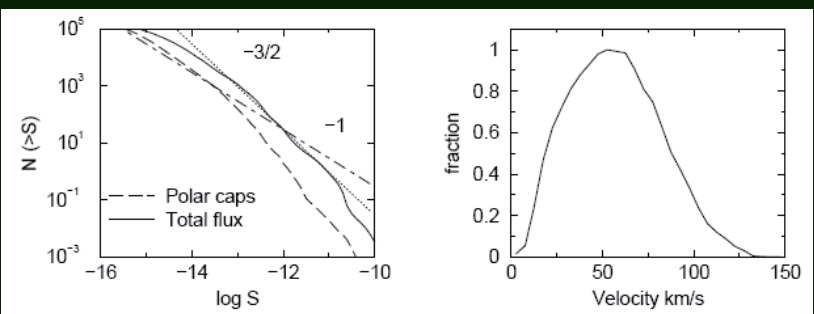

In the framework of a simplified model

(no subsonic propeller, no field decay,

no accretion inhibition, etc.) one can estimate properties of isolated accretors.
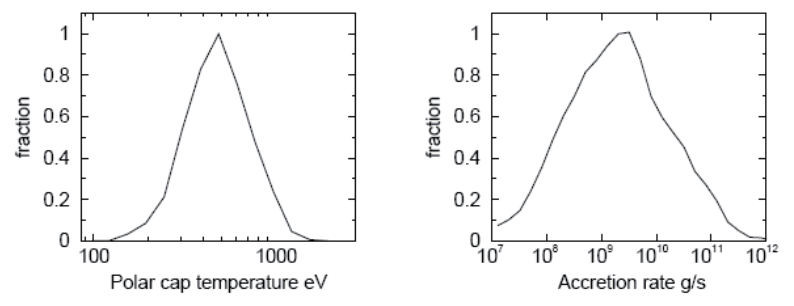

Slow, hot, dim, numerous at low fluxes $\left(<10^{-13} \mathrm{erg} / \mathrm{cm}^{2} / \mathrm{s}\right)$

Reality is more uncertain.

(astro-ph/0009225)

\section{Where and how to look for}

As sources are dim even in X-rays, and probably are extremely dim in other bands it is very difficult to find them.

In an optimistic scenario they outnumber cooling NSs at low fluxes.

Probably, for ROSAT they are to dim.

We hope that eROSITA will be able to identify accreting INSs.

Their spatial density at fluxes $\sim 10^{-15} \mathrm{erg} / \mathrm{cm}^{2} / \mathrm{s}$ is expected to be $\sim$ few per sq.degree in directions close to the galactic plane.

It is necessary to have an X-ray survey at $\sim 100-500 \mathrm{eV}$ with good resolution. 


\section{Magnetic field decay}

\section{Magnetic fields of NSs are expected to decay due to decay of currents which support them.}

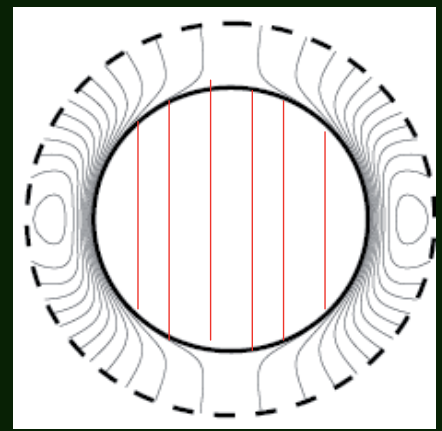

Crustal field of core field?

It is easy do decay in the crust.

In the core the filed is in the form

of superconducting vortices.

They can decay only when they are

moved into the crust (during spin-down).

Still, in most of model strong fields decay.

\section{Period evolution with field decay}

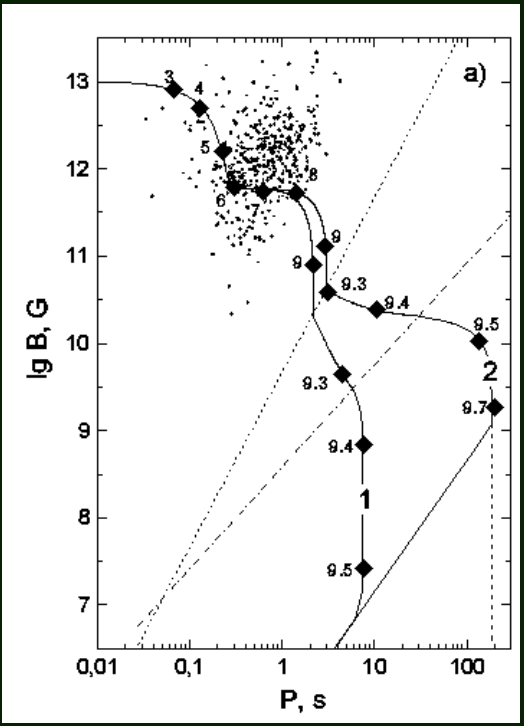

An evolutionary track of a NS is very different in the case of decaying magnetic field.

The most important feature is slow-down of spin-down. Finally, a NS can nearly freeze at some value of spin period.

Several episodes of relatively rapid field decay can happen.

Number of isolated accretors can be both decreased or increased in different models of field decay. But in any case their average periods become shorter and temperatures lower. 


\section{Magnetic field decay vs. thermal evolution}

\section{Magnetic field decay can be an important source of NS heating.}

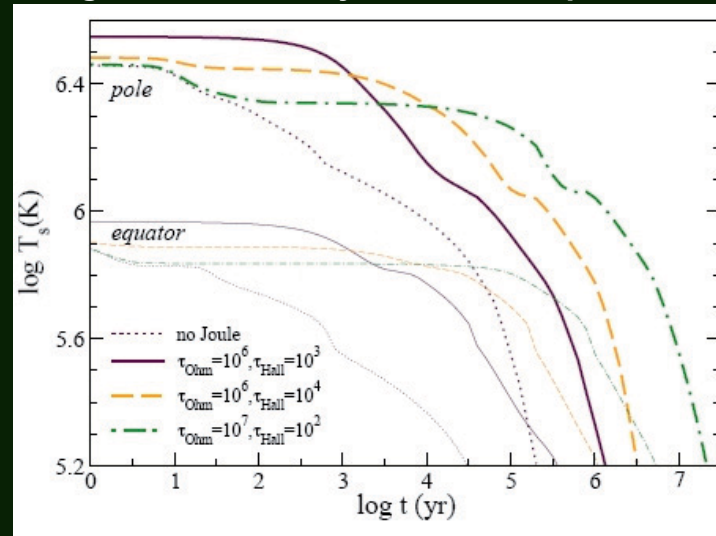

Heat is carried by electrons.

It is easier to transport heat along field lines. So, poles are hotter. (for light elements envelope the situation can be different).

Ohm and Hall decay

$$
B=B_{0} \frac{\exp \left(-t / \tau_{\mathrm{Ohm}}\right)}{1+\frac{\tau_{\mathrm{Ohm}}}{\tau_{\text {Hall }}}\left(1-\exp \left(-t / \tau_{\mathrm{Ohm}}\right)\right)}
$$

\section{Thermal heating for everybody?}

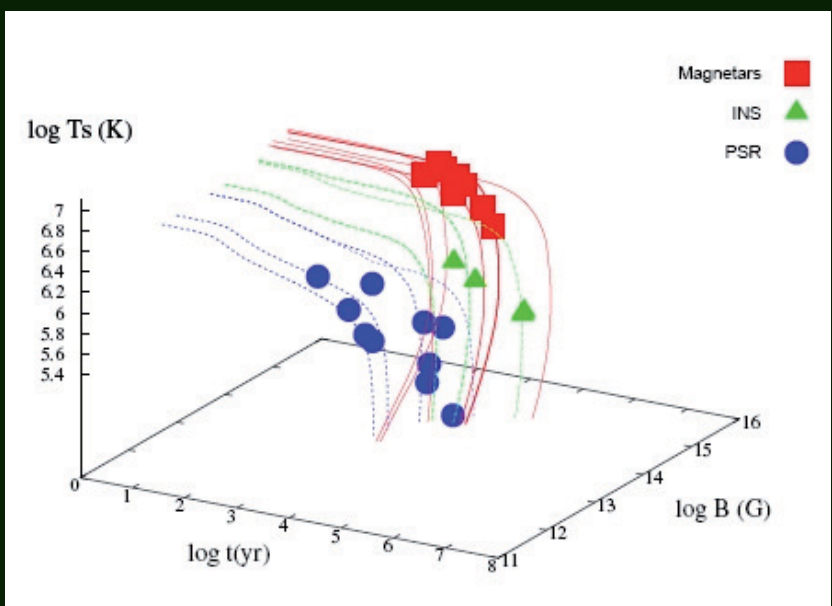

It is important to understand the role of heating by the field decay for different type. of INS.

In the model by Pons et al. the effect is more important for NSs with larger initial B.

Note, that the characteristic age estimate ( $p / 2$ pdot) are different in the case of decaying field!

arXiv: 0710.4914 (Aguilera et al.) 


\section{Magnetic field vs. temperature}

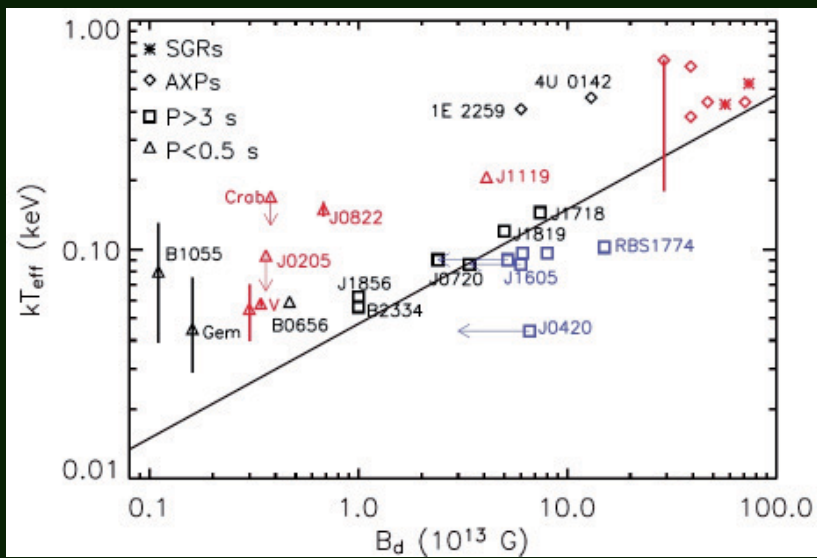

The line marks balance between heating due to the field decay and cooling. It is expected by the authors (Pons et al.) that a NS evolves downwards till it reaches the line, then the evolution proceeds along the line.

Selection effects are not well studied here. A kind of population synthesis modeling is welcomed.

(astro-ph/0607583)

\section{Radio pulsar braking: current losses}

The model of pulsar emission is not known, and also the model for spin-down is not known, too. Well-known magneto-dipole formula is just a kind of approximation.

One of competitors is longitudinal current losses model (Vasily Beskin et al.)

$$
\dot{P}=10^{-15} B_{12}^{10 / 7} P^{1 / 14} \cos ^{3 / 2} \chi .
$$

\section{Longitudinal current losses $\quad \underline{\text { Magneto-dipole }}$}

Both models predict evolution of the angle between spin and magnetic axis. Surprisingly, both are wrong!
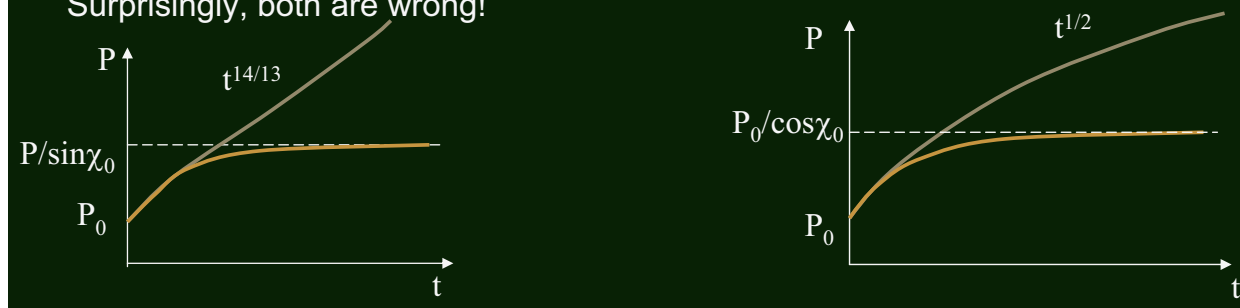


\section{Radio pulsar braking: braking index}

$n_{\mathrm{br}}=\frac{\Omega \ddot{\Omega}}{\dot{\Omega}^{2}} . \quad$ Braking index (definition)

$n_{\mathrm{br}}=3+2 \cot ^{2} \chi . \quad$ Magneto-dipole formula

$n_{\mathrm{br}}=1.93+1.5 \tan ^{2} \chi . \quad$ Longitudinal current losses

For well-measured braking indices $n<3$.

However, for many pulsars they are very large.

This can be simply an observational effect (microglitches, noise, etc.), but it can also be something real.

For example, related to the magnetic field evolution.

\section{Conclusions}

-We have some framework for spin evolution of NSs.

They are expected to passe several well-defined stages:

Ejector (including radion pulsar),

Propeller (probably, with subsonic substage),

Accretor.

NSs with large velocities (or fields) after the Ejector stage can appear as Georotators.

- In binaries we observe Ejectors, Propellers and Accretor.

For isolated NSs - only Ejectors (even, mostly radiopulsars).

- There are still many uncertainties related to the spin evolution:

1. Spin-down rate and angle evolution for radio pulsars

2. Subsonic propeller stage for isolated NSs

3. Inhibition of accretion at low rates

4. The role of the field decay 


\section{Conclusions-2}

- Observations of isolated accreting NSs can help a lot to understand all unknown questions of NS spin evolution and low-rate accretion.

- Magnetic field decay can be important also for young NSs, especially for highly magnetized ones, as a source of energy.

So, we have some coherent picture ..... But .....

A lot of funny thing a still waitng for us!

\section{Papers and books to read}

- Lupinov V.M. "Astrophysics of neutron stars"

- Lipunov, Postnov, Prokhorov "The Scenario Machine:

Binary Star Population Synthesis"

Astrophysics and Space Science Reviews (1996)

http://xray.sai.msu.ru/ mystery/articles/review/

- Popov et al. "The Neutron Star Census" ApJ 530, 896 (2000)

- Pons, Geppert "Magnetic field dissipation in neutron star crusts:

from magnetars to isolated neutron stars " astro-ph/0703267

- Ikhsanov "The origin of long-period X-ray pulsars" astro-ph/0611442 


\title{
Databases on NSs
}

1. ATNF. Pulsar catalogue http://www.atnf.csiro.au/research/pulsar/psrcat/

2. Magnetar database in McGill http://www.physics. mcgill.ca/ pulsar/magnetar/main.html

3. Be/X-ray binaries http://xray.sai.msu.ru/ raguzova/BeXcat/

\section{Lecture 3}

\section{Population synthesis}

\author{
Sergei Popov (SAI MSU)
}

Dubna "Dense Matter In Heavy lon Collisions and Astrophysics", July 2008 


\section{Population synthesis in astrophysics}

A population synthesis is a method of a direct modeling of relatively large populations of weakly interacting objects with non-trivial evolution.

As a rule, the evolution of the objects is followed from their birth up to the present moment.

see astro-ph/0411792

$$
\text { and }
$$

Physics-Uspekhi 50, 1123

(УФН 2007 г., N11; http://www.ufn.ru)

\section{Why PS is necessary?}

1. No direct experiments $\square$ computer experiments

2. Long evolutionary time scales

3. Selection effects. We see just a top of an iceberg.

4. Expensive projects for which it is necessary to make predictions 


\section{Tasks}

1. To test and/or to determine initial and evolutionary parameters.

To do it one has to compare calculated and observed populations.

This task is related to the main pecularity of astronomy:

we cannot make direct experiments under controlled conditions.

2. To predict properties of unobserved populations.

Population synthesis is actively used to define programs for future observational projects: satellites, telescopes, etc.

\section{Two variants}

\section{Evolutionary and Empirical}

1. Evolutionary PS.

The evolution is followed from some early stage.

Typically, an artificial population is formed

(especially, in Monte Carlo simulations)

2. Empirical PS.

It is used, for example, to study integral properties

(spectra) of unresolved populations.

A library of spectra is used to predict integral properties. 


\section{Examples}

1. PS of radiopulsars

2. PS of gamma-ray pulsars

3. PS of close-by cooling NSs

4. PS of isolated NSs

5. PS of close binary systems

\section{Population synthesis of radio pulsars}

The idea was to make an advance population synthesis study of normal radio pulsar to reproduce the data observed in PMBPS and Swinburne. Comparison between actual data and calculations should help to understand better the underlying parameteres and evolution laws.

Only normal (non-millisecond, non-binary, etc.) pulsars are considered.

Note, however, that the role of pulsars originated in close binaries can be important.

\section{Ingredients}

- Velocity distribution

- Spatial distribution

- Galactic model

- Initial period distribution

- Initial magnetic field distribution

- Field evolution (and angle)

- Radio luminosity

- Dispersion measure model

- Modeling of surveys
The observed PSR sample is heavily biased. It is necessary to model the process of detection, i.e. to model the same surveys in the synthetic Galaxy.

A synthetic PSR is detected if it appears in the area covered by on pf the survey, and if its radio flux exceeds some limit.

2/3 of known PSRs were detected in PBMPS or/and SM (914 and 151).

(following Faucher-Giguere and Kaspi astro-ph/0512585) 


\section{Velocity distribution}

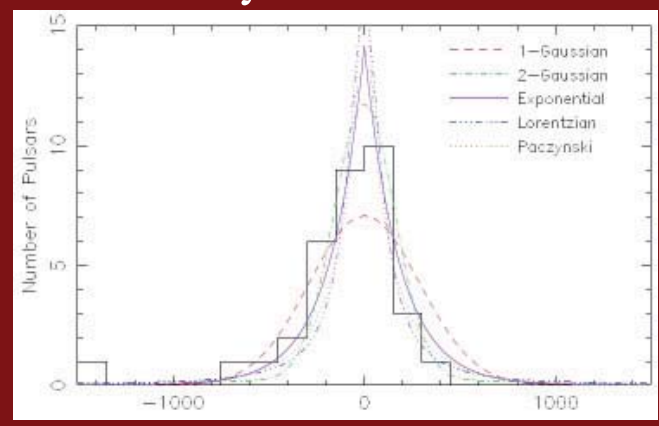

Observational data for 34 PSRs. $\mathrm{V}_{\max }=1340 \mathrm{~km} / \mathrm{s}$ (PSR B2011+38).

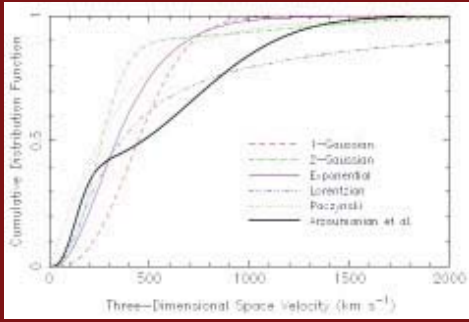

The authors checked different velocity distributions: single maxwellian, double maxwellian, loretzian, paczynski mode, and double-side exponential. The last one was takes for the reference model. Single maxwellian was shown to be inadequate.

$$
p\left(v_{l}\right)=\frac{1}{2\left\langle v_{l}\right\rangle} \exp \left(-\frac{\left|v_{l}\right|}{\left\langle v_{l}\right\rangle}\right),
$$

$v_{q}=D \mu_{l} \cos b-\Delta y_{y}$ is the component of the pulsar's transverse velocity parallel to the plane, relative to itx LSR, where $\Delta v_{2}(D, I, b)$ is the contribution to the observer velocity due to differential Galactic rotation and the motion of the Sun relative to its own LSR

\section{Spatial distribution}

Initial spatial ditribution of PSRs was calculated in a complicated realistic way.

- exponential dependences ( $R$ and $Z$ ) were taken into account

- Spiral arms were taken into account

- Decrease of PSR density close to the Galactic center was used

\begin{tabular}{ccccc}
\hline \hline Arm Number & Name & $\begin{array}{c}k \\
(\mathrm{rad})\end{array}$ & $\begin{array}{c}r_{0} \\
(\mathrm{kpc})\end{array}$ & $\begin{array}{c}\theta_{0} \\
(\mathrm{rad})\end{array}$ \\
\hline 1 & Norma & 4.25 & 3.48 & 1.57 \\
2 & Carina-Sagittarius & 4.25 & 3.48 & 4.71 \\
3 & Perseus & 4.89 & 4.90 & 4.09 \\
4 & Crux-Scutum & 4.89 & 4.90 & 0.95 \\
\hline
\end{tabular}

$\theta(r)=k \ln \left(r / r_{0}\right)+\theta_{0}$.

However, some details are still missing. For example, the pattern is assumed to be stable during all time of calculations (i.e. corotating with the Sun).

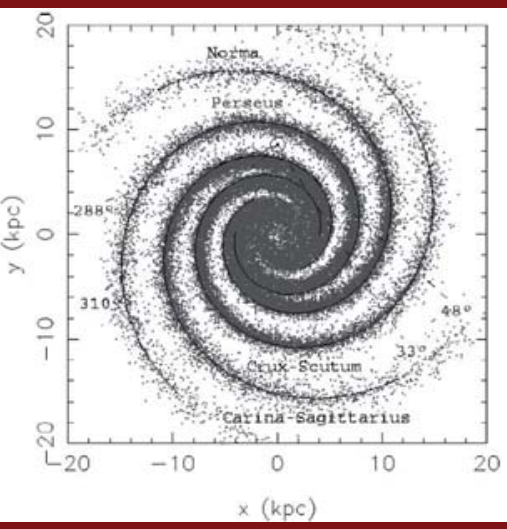




\section{Galactic potential}

The potential was taken from Kuijken and Gilmore (1989):

- disc-halo

- buldge

- nuclei

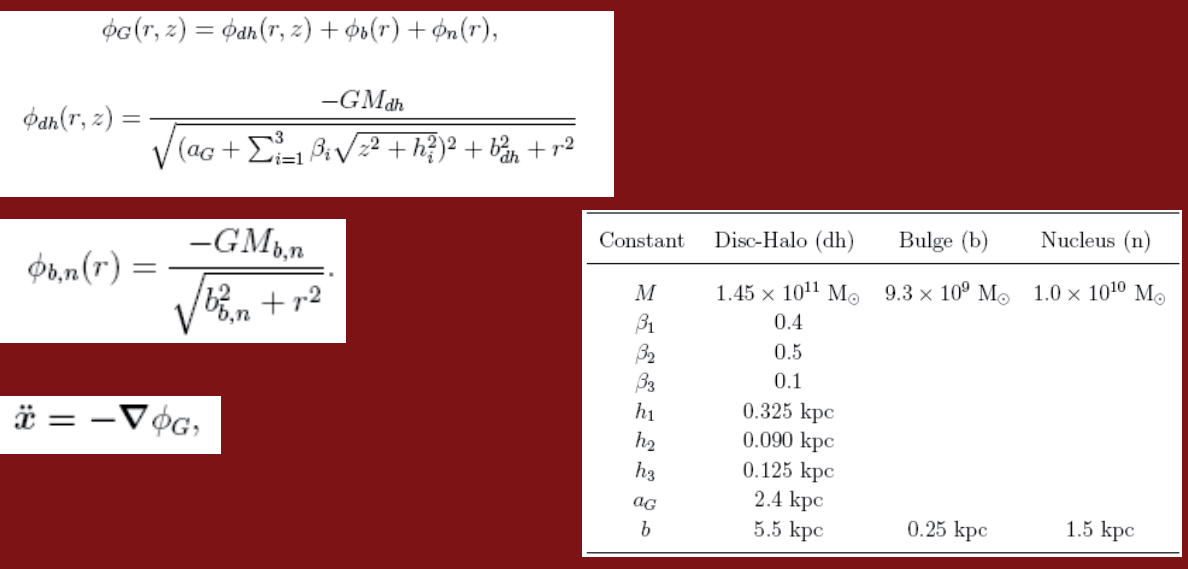

\section{Initial spin periods and fields}

Spin periods were randomly taken from a normal distribution. Magnetic fields - also from a normal distribution for log B.

The authors do not treat separately the magnetic field and inclination angle evolution.

Purely magneto-dipole model with $\mathrm{n}=3$ and $\sin \mathrm{X}=1$ is used.

$R_{\mathrm{NS}}=10^{6} \mathrm{~cm}, \mathrm{l}=10^{45}$.

$$
P \dot{P}=\left(\frac{8 \pi^{2} R^{6}}{3 I c^{3}}\right) B^{2} \sin ^{2} \chi, \quad \mathrm{P} \sim\left(\mathrm{P}^{2}{ }_{0}+\mathrm{K} \mathrm{t}\right)^{1 / 2}
$$

The death-line is taken in the usual form:

$$
\frac{B}{P^{2}}=0.17 \times 10^{12} \mathrm{G} \mathrm{s}^{-2}
$$




\section{Radio luminosity and beaming}

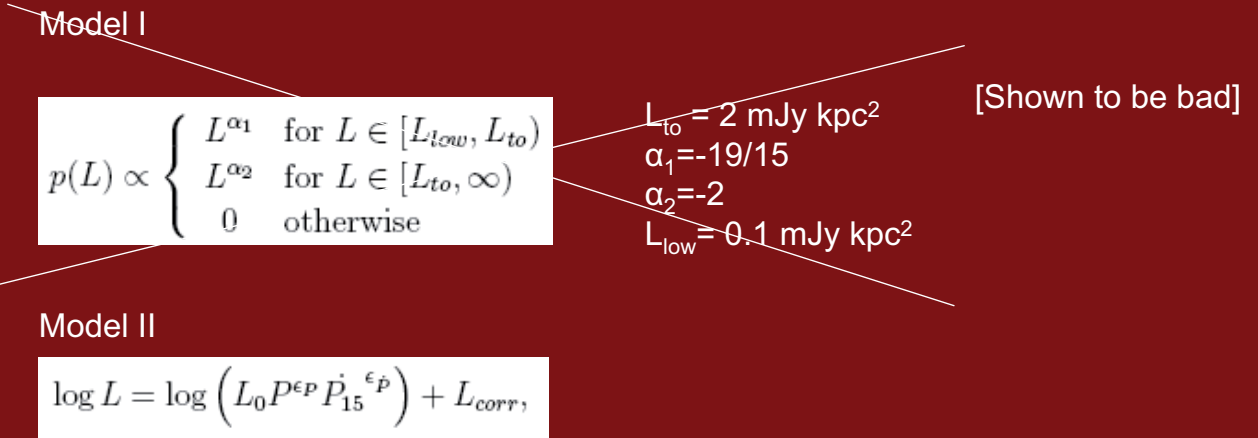

\section{Optimal model and simulations}

\begin{tabular}{cc} 
Model Parameter & Value \\
\hline Radial Distribution Model & Yusifov \& Küçük \\
$R_{1}$ & $0.55 \mathrm{kpc}$ \\
$a$ & 1.64 \\
$b$ & 4.01 \\
\hline Birth Height Distribution & Exponential \\
$\left\langle z_{0}\right\rangle$ & $50 \mathrm{pc}$ \\
\hline Birth Velocity Distribution & Exponential \\
$\left\langle v_{3 D}\right\rangle$ & $380 \mathrm{~km} \mathrm{~s}{ }^{-1}$ \\
\hline Birth Spin Period Distribution & Normal \\
$\left\langle P_{0}\right\rangle$ & $300 \mathrm{~ms}$ \\
$\sigma_{P_{0}}$ & $150 \mathrm{~ms}$ \\
\hline Magnetic Field Distribution & Log-Normal \\
$\langle\log (B / \mathrm{G})\rangle$ & 12.65 \\
$\sigma_{\log B}$ & 0.55 \\
\hline Luminosity Model & $P-P$ Power Paw \\
$L_{0}$ & $0.18 \mathrm{mJy} \mathrm{kpc}^{2}$ \\
$\epsilon_{P}$ & -1.5 \\
$\epsilon_{\dot{P}}$ & 0.5 \\
$\sigma_{L_{c o r r}}$ & 0.8 \\
\hline
\end{tabular}

The code is run till the number of "detected" synthetic PSR becomes equal to the actual number of detected PSRs in PBMPS and SM.

\section{For each simulation the "observed"} distributions of $b, I, D M, S_{1400}, P$, and $B$, are compared with the real sample.

It came out to be impossible to to apply only statistical tests.

Some human judgement is necessary for interpretation. 


\section{Results}

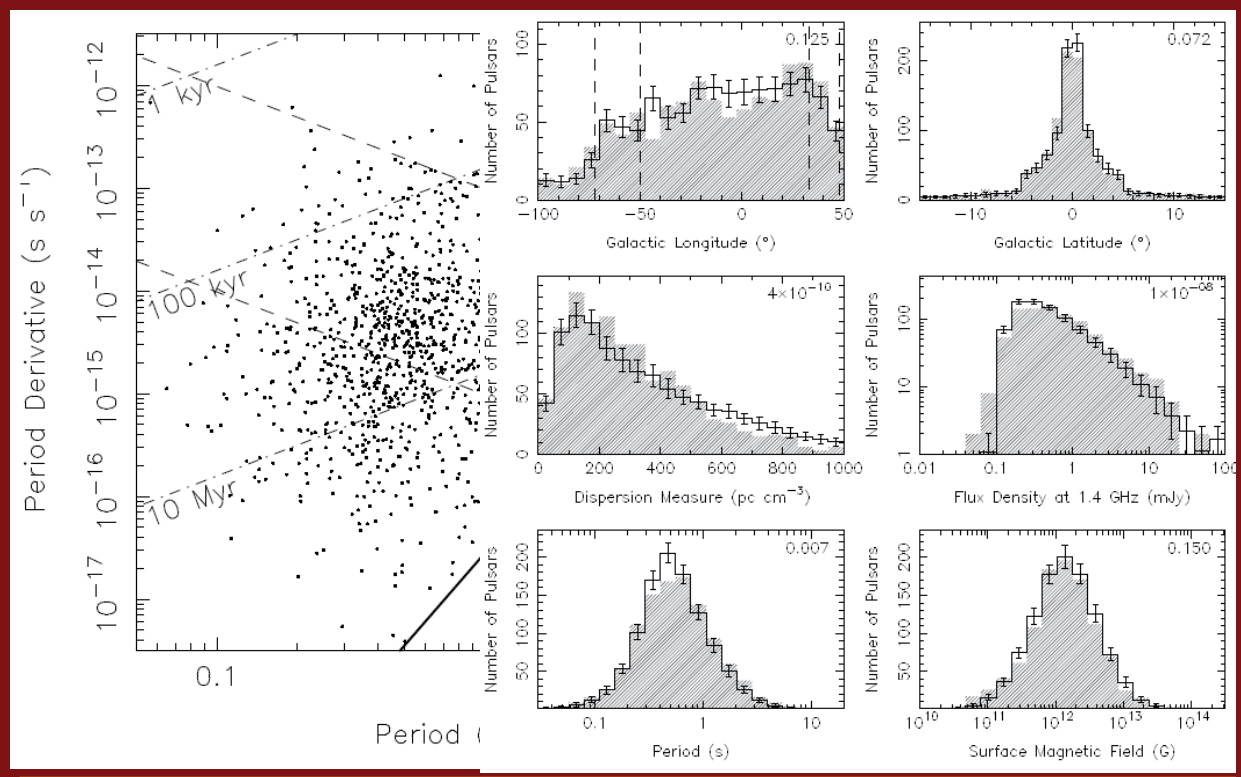

Solid lines - calculation, hatched diagrams - real observations

\section{Discussion of the results}

1. No significant field decay (or change in the inclination angle) is necessary to explain the data.

2. Results are not very sensitive to braking index distribution

3. Birthrate is $2.8+/-0.1$ per century.

If between $13 \%$ and $25 \%$ of core collapse SN produce BHs, then there is no necessity to assume a large population of radio quiet NSs.

120000 PSRs in the Galaxy 


\section{Population synthesis of gamma-ray PSRs}

\section{Ingredients}

1. Geometry of radio and gamma beam

2. Period evolution

3. Magnetic field evolution

4. Initial spatial distribuion

5. Initial velocity distribution

6. Radio and gamma spectra

7. Radio and gamma luminosity

8. Properties of gamma detectors

9. Radio surveys to comapre with.

\section{Tasks}

1. To test models

2. To make predictions for GLAST and AGILE

(following Gonthier et al astro-ph/0312565)

\section{Population of close-by young NSs}

- Magnificent seven

- Geminga and 3EG J1853+5918

- Four radio pulsars with thermal emission (B0833-45; B0656+14; B1055-52; B1929+10)

- Seven older radio pulsars, without detected thermal emission.

To understand the origin of these populations and predict future detections it is necessary to use population synthesis. 
Population synthesis: ingredients

- Birth rate of NSs

- Initial spatial distribution

- Spatial velocity (kick)

- Mass spectrum

- Thermal evolution

- Interstellar absorption

- Detector properties

\section{Task:}

To build an artificial model

of a population of some astrophysical sources and

to compare the results of calculations with observations.

\section{Population synthesis - I.}

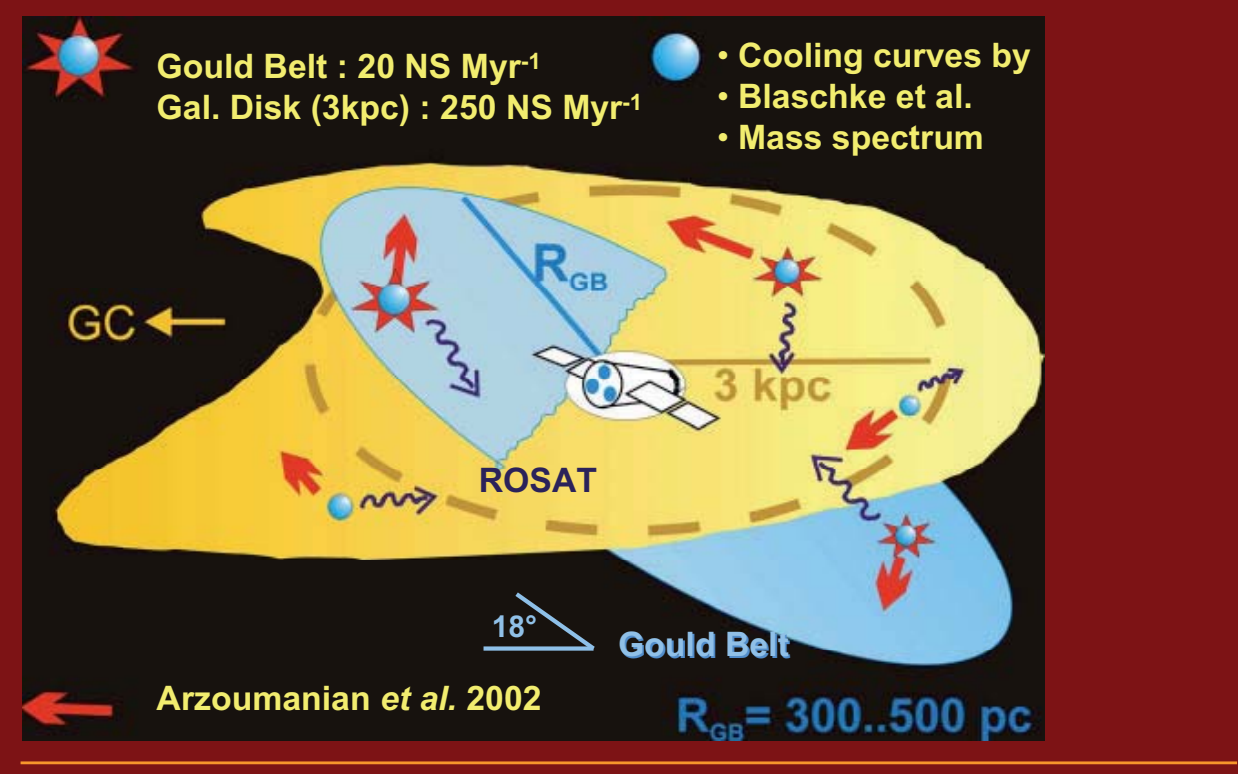




\section{The Gould Belt}

- Poppel (1997)

- $\mathrm{R}=300-500 \mathrm{pc}$

- Age 30-50 Myrs

- Center at 150 pc from the Sun

- Inclined respect to the galactic plane at 20 degrees

- 2/3 massive stars in 600 pc belong to the Belt

- 20-30 SN per Myr
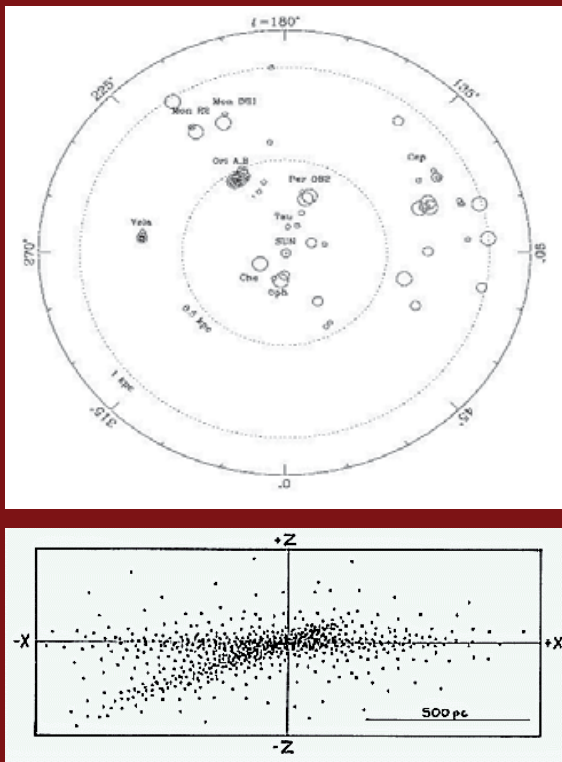

Mass spectrum of $\mathrm{NSs}$

- Mass spectrum of local young NSs can be different from the general one (in the Galaxy)

- Hipparcos data on near-by massive stars

- Progenitor vs NS mass: Timmes et al. (1996); Woosley et al. (2002)

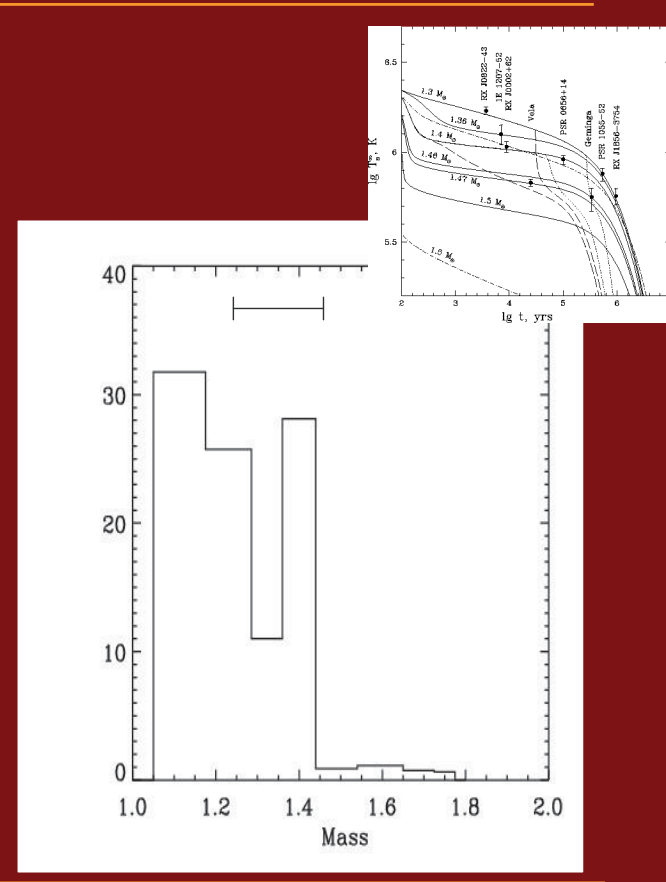




\section{Progenitor mass vs. NS mass}

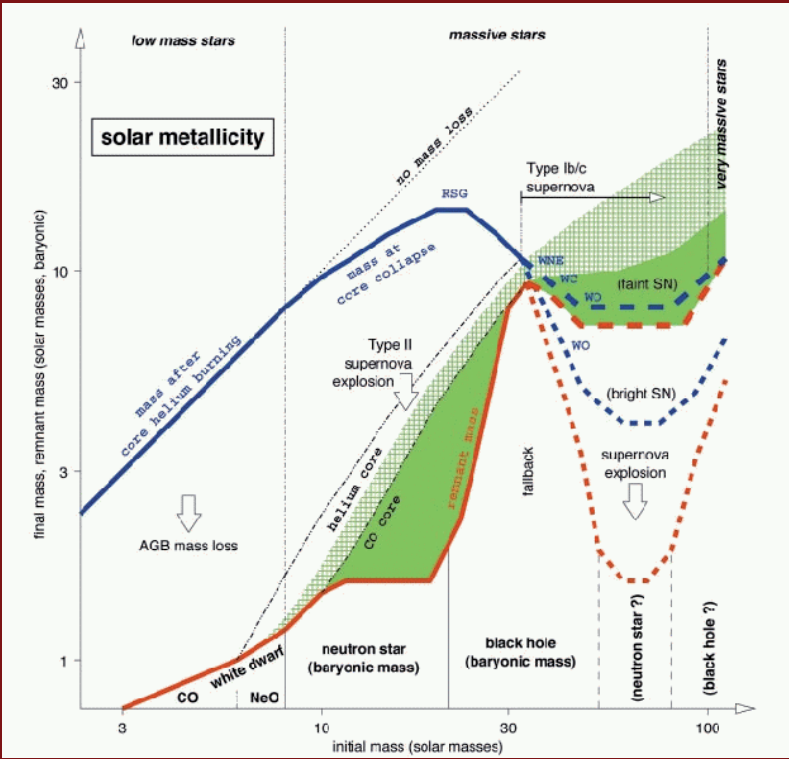

Woosley et al. 2002

\section{Cooling of NSs}

- Direct URCA

- Modified URCA

- Neutrino bremstrahlung

- Superfluidity

- Exotic matter (pions,

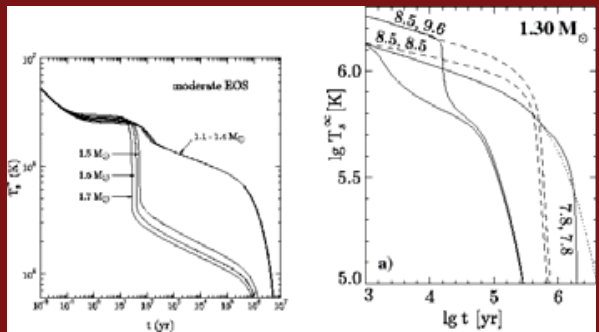
quarks, hyperons, etc.)

In our study we use a set of cooling curves calculated by Blaschke, Grigorian and Voskresenski (2004) in the frame of the Nuclear medium cooling model 


\section{$\log N-\log S$}

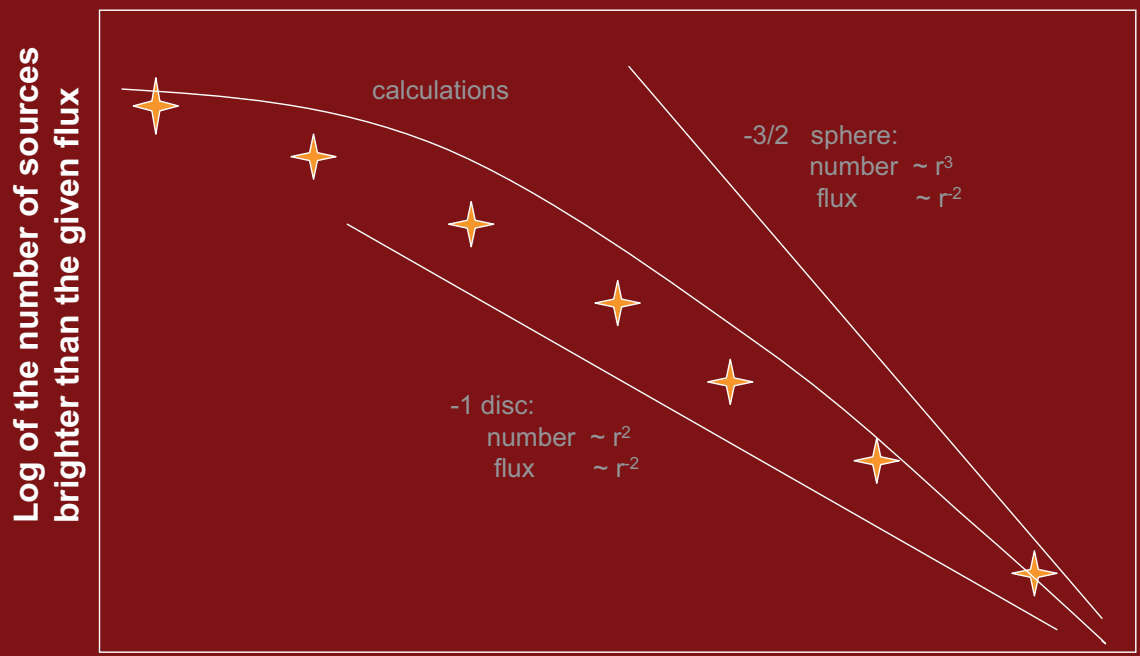

Log of flux (or number counts)

\section{Some results of PS-I:}

\section{$\log N-\log S$ and spatial distribution}
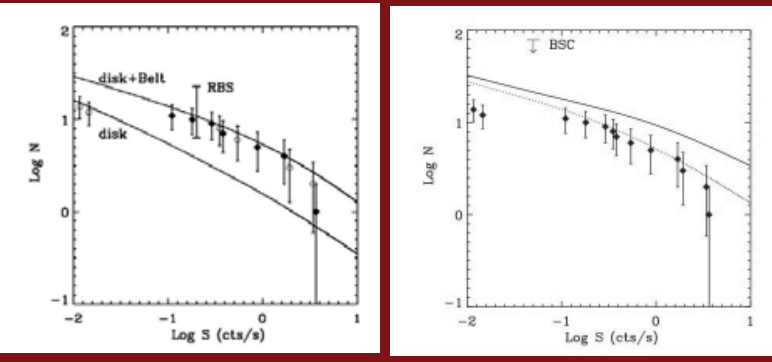

Log N - Log S for closeby ROSAT NSs can be explained by standard cooling curves taking into account the Gould Belt.

More than $1 / 2$ are in +/- 12 degrees from the galactic plane.

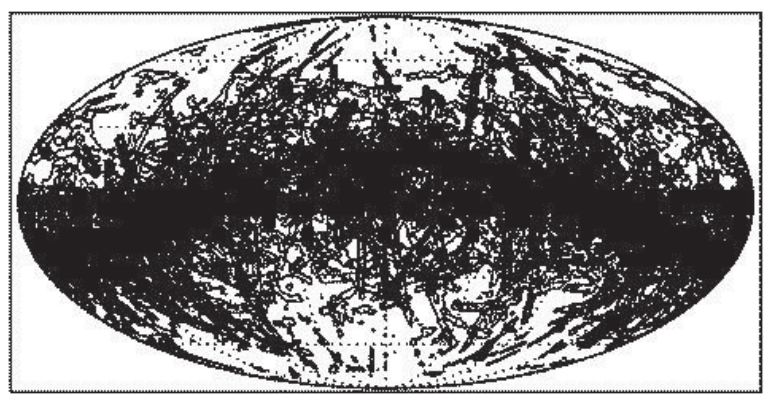
$19 \%$ outside $+/-30^{\circ}$ $12 \%$ outside $+/-40^{\circ}$

(Popov et al. 2005 Ap\&SS 299, 117) 


\section{Population synthesis - II. recent improvements}

\section{Spatial distribution of progenitor stars}

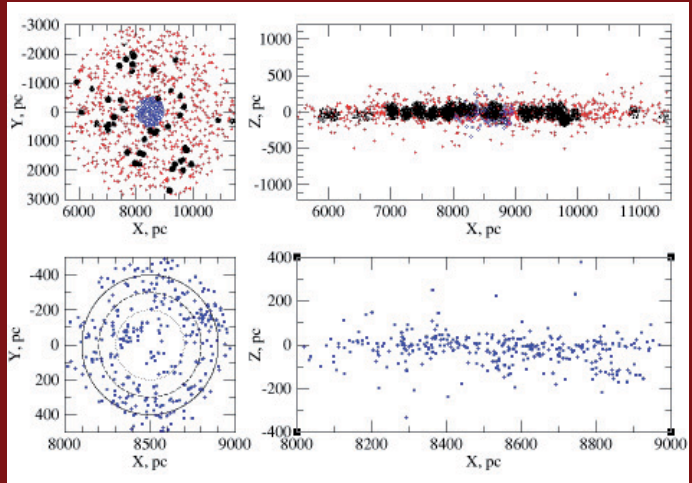

a) Hipparcos stars up to 500 pc

[Age: spectral type \& cluster age (OB ass)]

b) 49 OB associations: birth rate $\sim N_{\text {star }}$

c) Field stars in the disc up to $3 \mathrm{kpc}$
We use the same

normalization for

NS formation rate

inside 3 kpc: 270 per Myr.

Most of NSs are born in

$\mathrm{OB}$ associations.

For stars $<500$ pc we even try to take into account if they belong to $\mathrm{OB}$ assoc. with known age.

\section{Population synthesis - II.}

recent improvements

\section{Spatial distribution of ISM $\left(\mathbb{N}_{\mathrm{H}}\right)$}

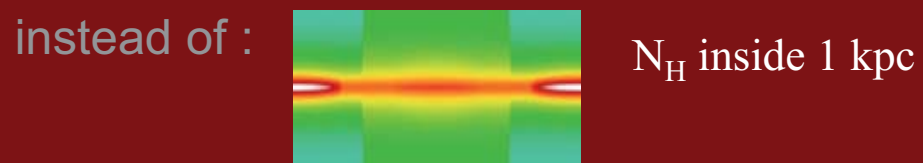

now:

(see astro-ph/0609275 for details)

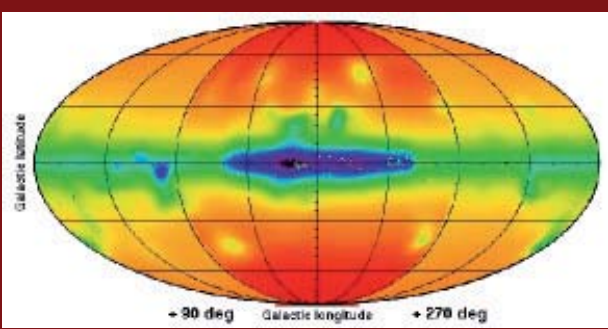

Modification of the old one

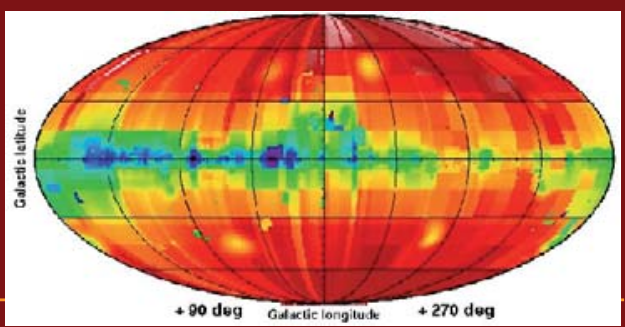

Hakkila 


\section{0 tracks, new ISM model}

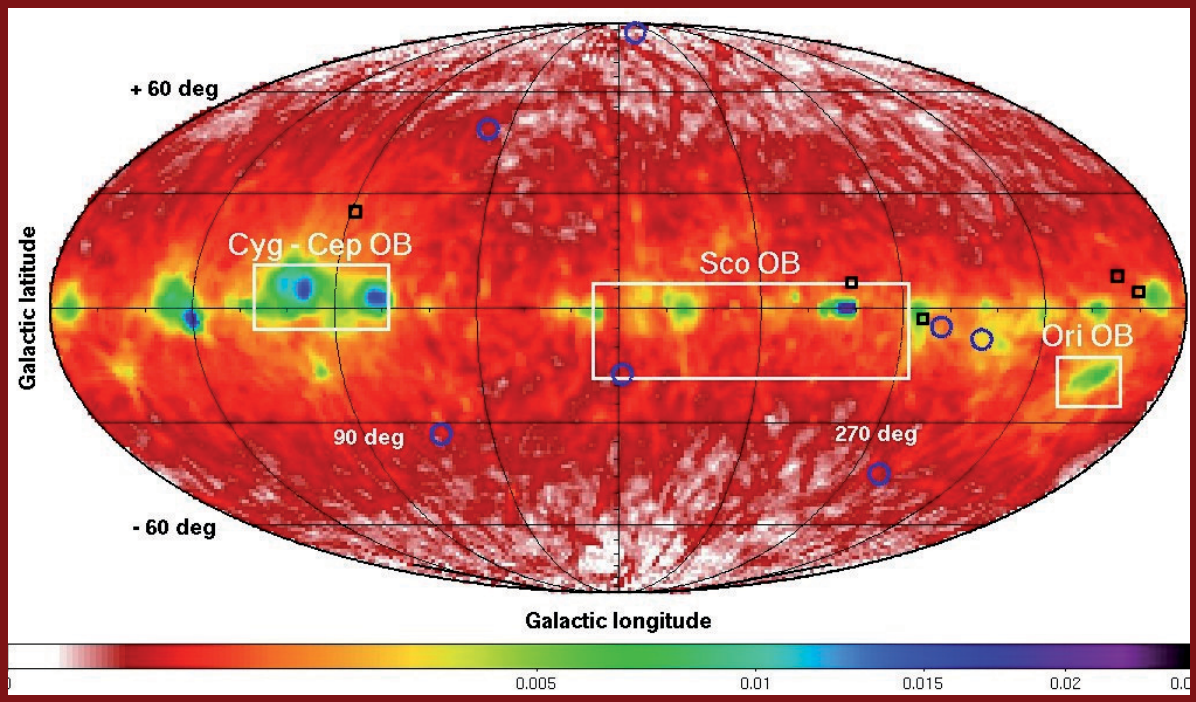

Predictions for future searches

(Posselt et al. arXiv: 0801.4567)

$\log \mathrm{N}-\log \mathrm{S}$ as an additional test

- Standard test: Age - Temperature

$\square$ Sensitive to ages $<10^{5}$ years

$\square$ Uncertain age and temperature

$\square$ Non-uniform sample

- $\log N-\log S$

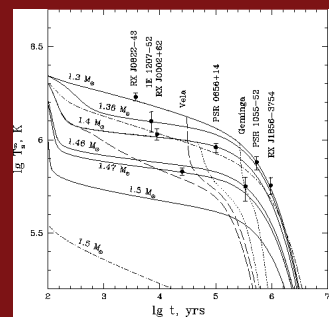

$\square$ Sensitive to ages $>10^{5}$ years (when applied to close-by NSs) $\square$ Definite N (number) and S (flux)

$\square$ Uniform sample

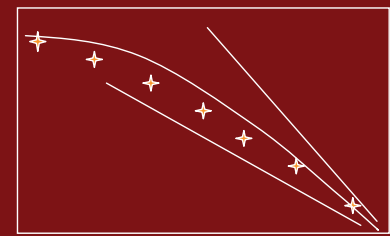

- Two test are perfect together!!! 


\section{Model I}

- Pions.

- Gaps from Takatsuka \& Tamagaki (2004)

- $\mathrm{T}_{\mathrm{s}}-\mathrm{T}_{\text {in }}$ from Blaschke, Grigorian, Voskresenky (2004)
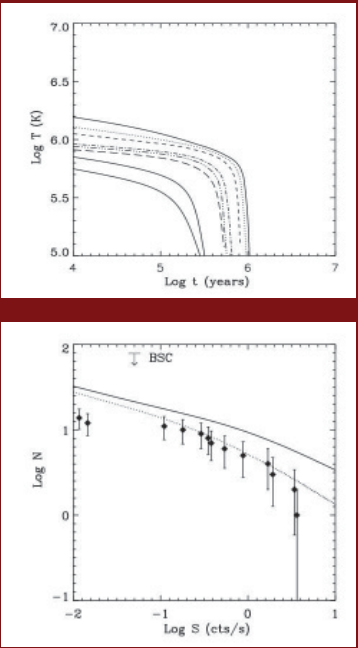

\section{Model II}

- No Pions

- Gaps from Yakovlev et al. (2004), ${ }^{3} P_{2}$ neutron gap suppressed by 0.1

- $\mathrm{T}_{\mathrm{s}}-\mathrm{T}_{\text {in }}$ from Tsuruta (1979)
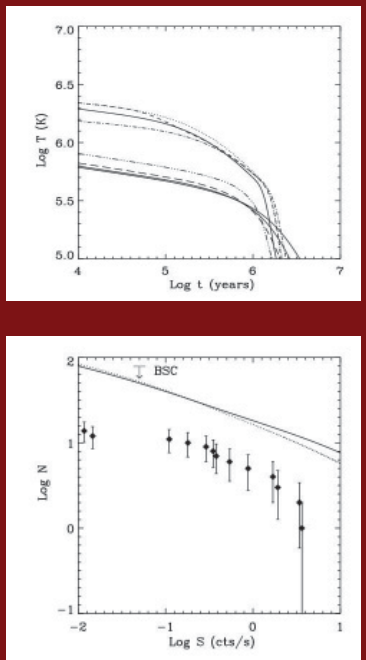
Sensitivity of $\log N-\log S$

- $\log N-\log S$ is very sensitive to gaps

- $\log N-\log S$ is not sensitive to the crust if it is applied to relatively old objects $\left(>10^{4-5} \mathrm{yrs}\right)$

- $\log N-\log S$ is not very sensitive to presence or absence of pions

We conclude that the two test complement each other

Results for HySs application

\begin{tabular}{|l||c|c||c|c|c|c|c|}
\hline Model & $\Delta_{0}[\mathrm{MeV}]$ & $\alpha$ & $\mathrm{BC}$ & $\mathrm{T}-\mathrm{t}$ & $\log \mathrm{N}-\mathrm{Log} \mathrm{S}$ & $\mathrm{M}_{\text {typ }} \leq 1.5 \mathrm{M}_{\odot}$ & All tests \\
\hline \hline I & 1 & 10 & + & + & $\circ$ & - & - \\
II & 0.1 & 0 & + & - & + & - & - \\
III & 0.1 & 2 & + & $\circ$ & + & - & - \\
IV & 5 & 25 & + & + & + & + & + \\
\hline
\end{tabular}

One model among four was able to pass all tests.
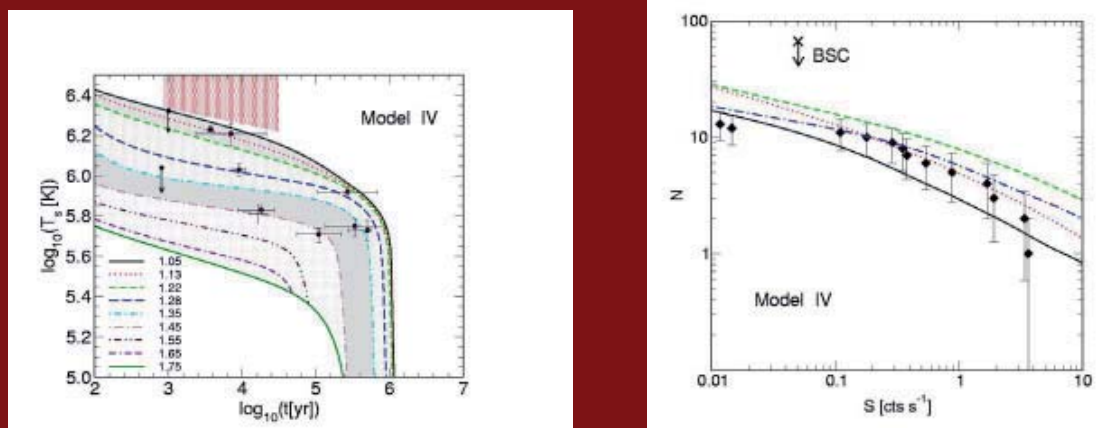


\section{Isolated neutron star census}

\section{Task.}

- To calculate distribution of isolated NSs in the Galaxy over evolutionary stages:

Ejector, Propeller, Accretor, Georotator

- Predict the number of accretors

\section{Ingredients.}

- Galactic potential

- Initial NS spatial distribution

- Kick velocity

- ISM distribution

- Spin initial distribution, evolution and critical periods

- Magnetic field initial distribution and evolution

\section{Stages}
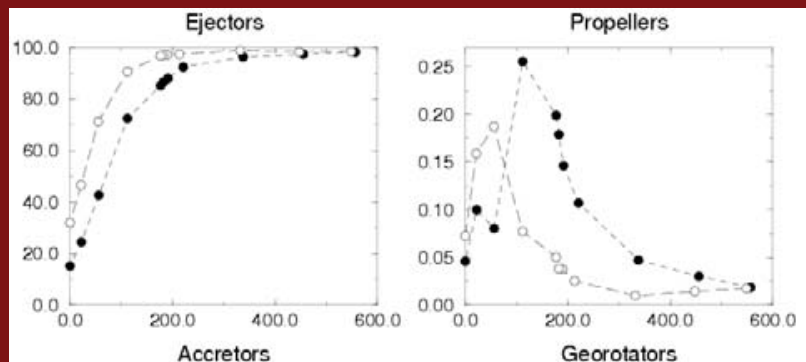

Rather conservative evolutionary scheme was used.

For example, supersonic propellers have not been considered (Ikhsanov 2006).
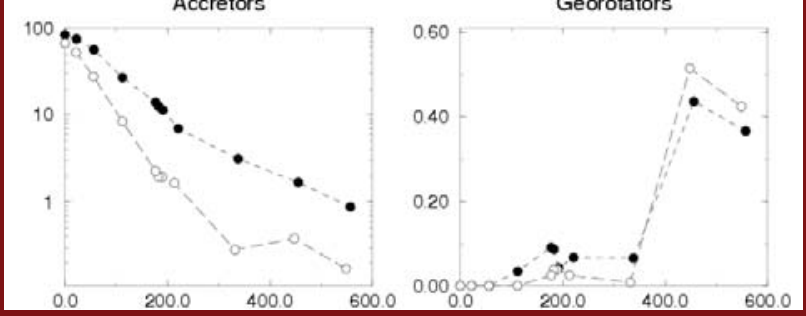

astro-ph/9910114 


\section{Accreting isolated NSs}

At small fluxes $<10^{-13} \mathrm{erg} / \mathrm{s} / \mathrm{cm}^{2}$ accretors can become more abundant than coolers. Accretors are expected to be slightly harder: 300-500 eV vs. 50-100 eV. Good targets for eROSITA!

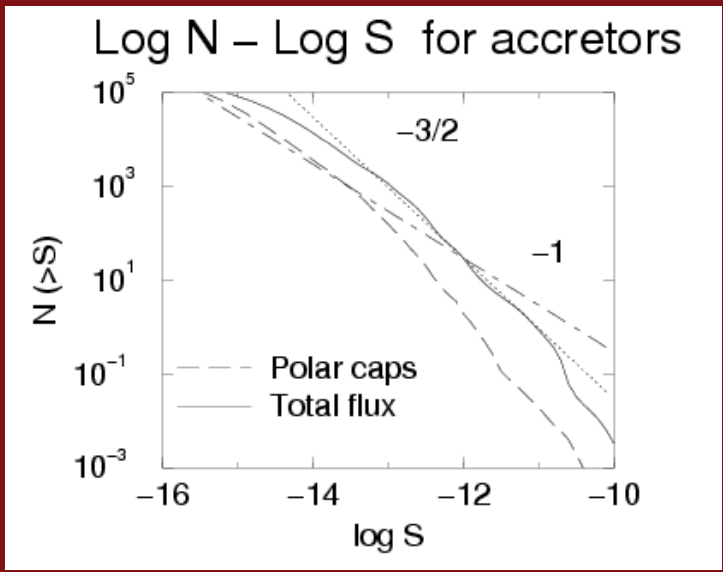

From several hundreds up to several thousands objects at fluxes about few $\times 10^{-14}$, but difficult to identify.

Monitoring is important.

Also isolated accretors can be found in the Galactic center (Zane et al. 1996, Deegan, Nayakshin 2006).

\section{Population synthesis of binary systems}

Interacting binaries are ideal subject for population synthesis studies:

- The are many of them observed

- Observed sources are very different

- However, they come from the same population of progenitors...

-... who's evolution is non-trivial, but not too complicated.

- There are many uncertainties in evolution ...

-... and in initial parameters

- We expect to discover more systems

-... and more types of systems

- With new satellites it really happens! 


\section{Scenario machine}

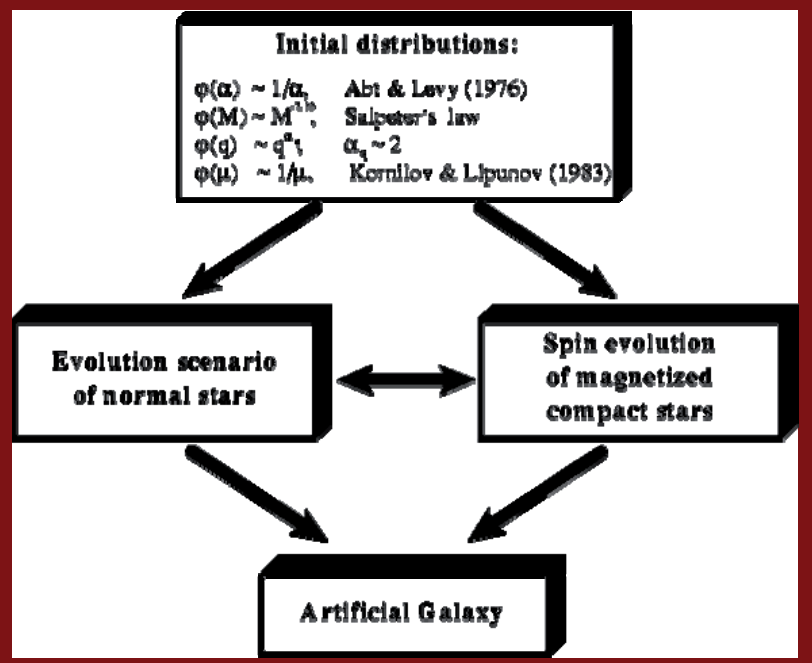

There are several groups

in the world which study

evolution of close binaries

using population synthesis

approach.

\section{Examples of topics}

- Estimates of the rate of coalescence of NSs and BHs

- X-ray luminosities of galaxies

- Calculation of mass spectra of NSs in binaries

- Calculations of SN rates

- Calculations of the rate of short GRBs

\section{Evolution of close binaries}
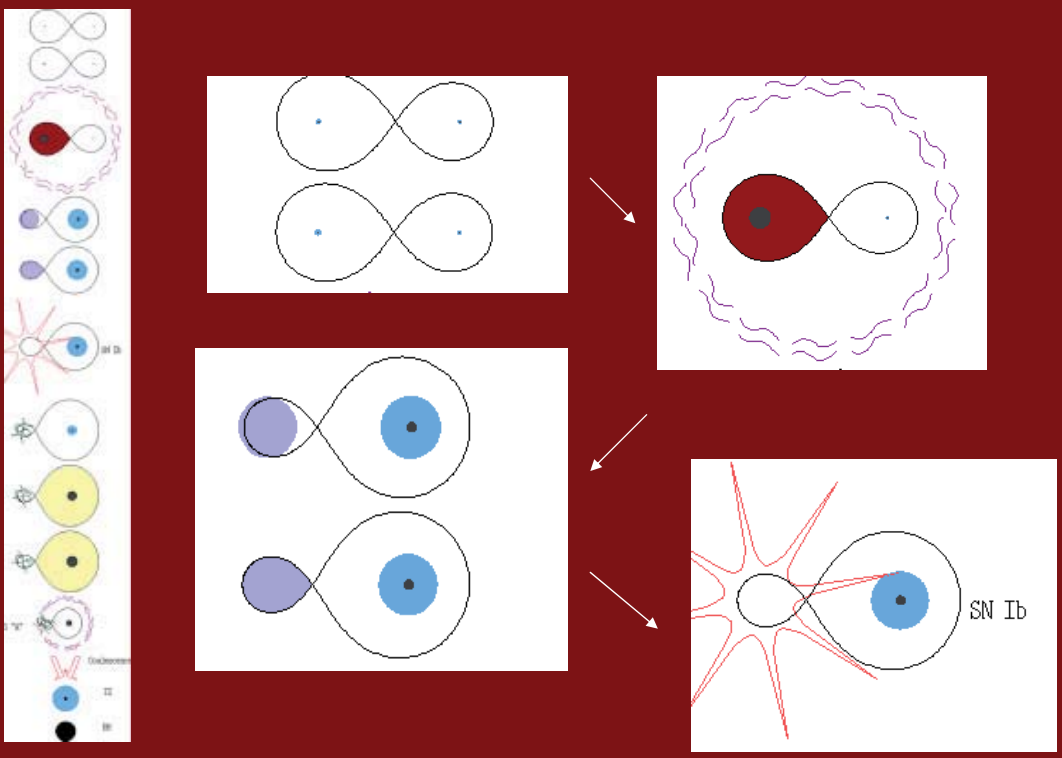


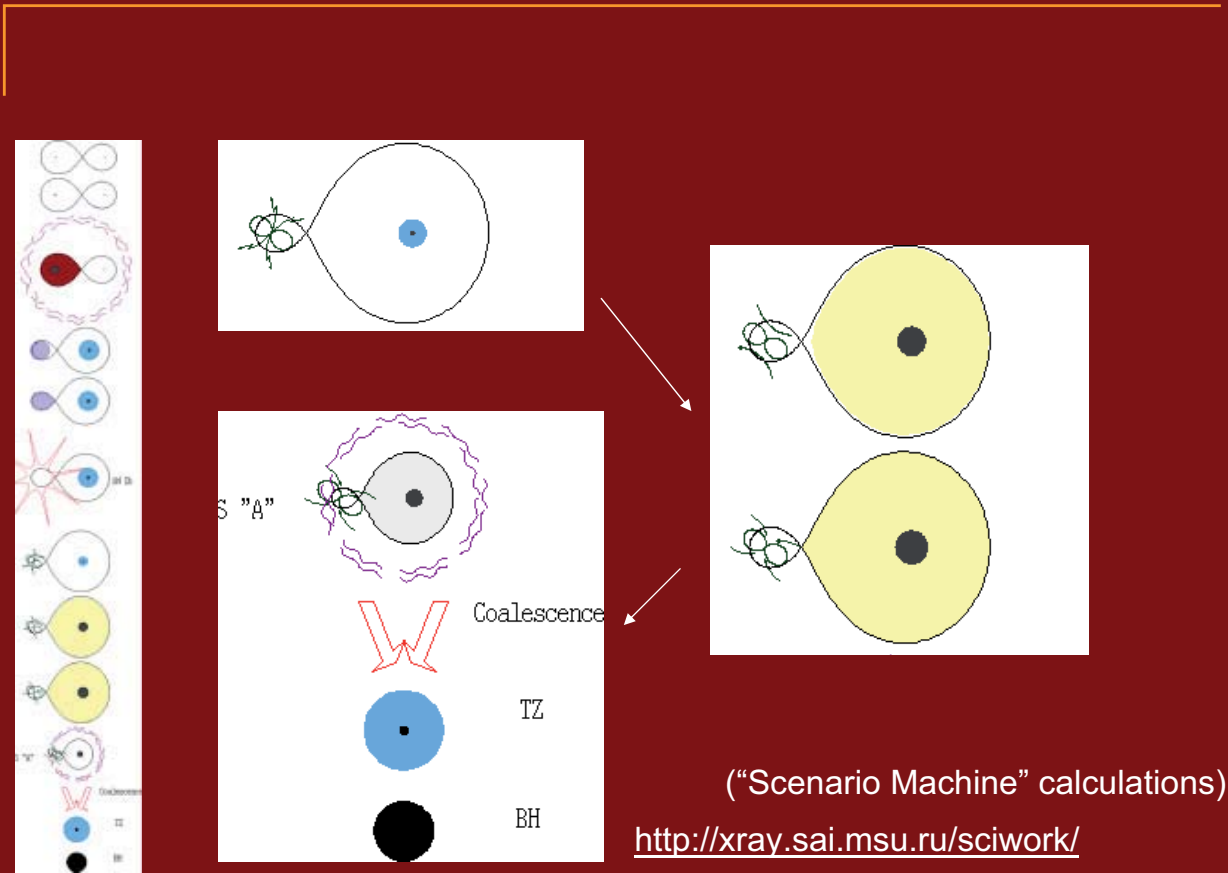

(«Вокруг света» июль 2008 г. http://vokrugsveta.ru)

\section{Looking for new magnetars}

There are many archival XMM-Ney Why not to use them to search for How?

Just using the fact that all known m
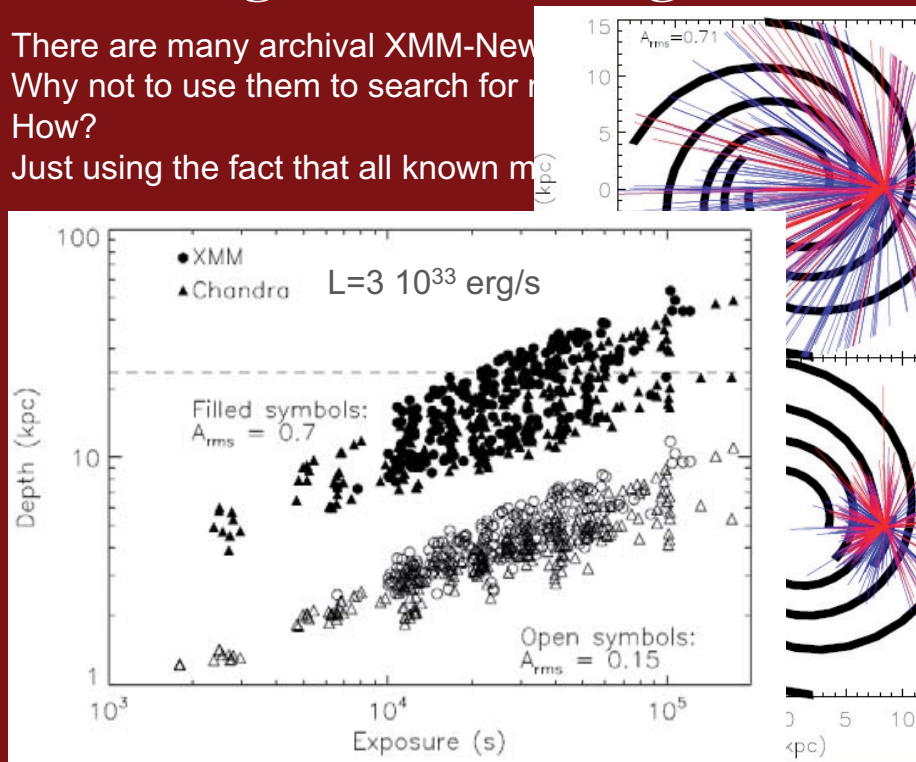

ations.

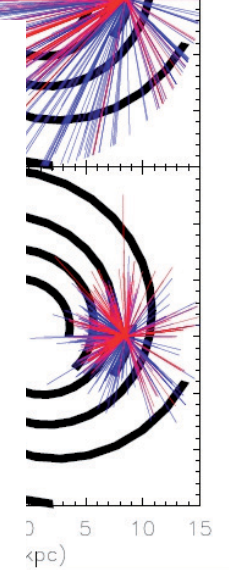

narrow range!

s of the

magnetars. $\leqslant 100$ or $<500$.

(0711.0988)

By the way, they also can put contraints on M7-like sources..... 


\section{Looking for new M7-like sources}

M7-like objects are very interesting by themselves and are important for studies of NS physics.

Several campains have been made to look for more sources.

- Agueros et al. (astro-ph/0511659)

- Chieregato et al. (astro-ph/0502292)

Looking for blank field soft X-rays sources (extreme $\mathrm{f}_{\mathrm{x}} / \mathrm{f}_{\mathrm{opt}}$ ratio).

Chieregato et al. searched for blank field sources with the ROSAT HRI data (only $\sim 1.8 \%$ of the sky, mostly at high galactic latitudes).

Several candidates have been figured out.

Agueros et al. used ROSAT All-sky Survey and SDSS.

Also several candidates have been found.

\section{Predictions for future searches and candidates}

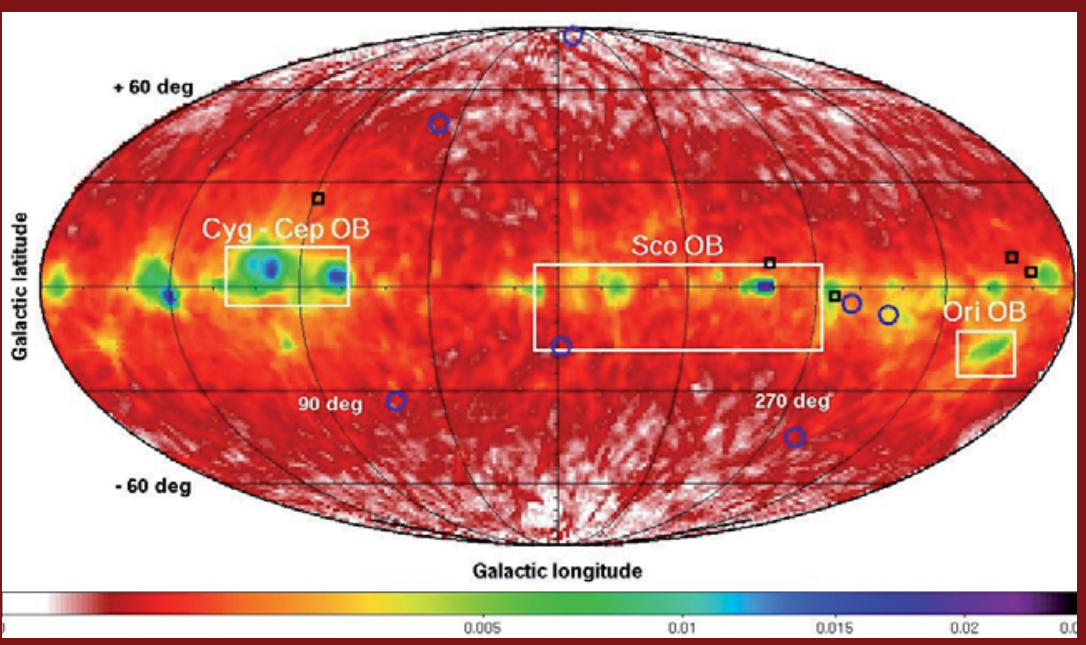

(Posselt et al. arXiv: 0801.4567) 


\section{Looking for isolated accretors}

Many programs aimed to find accreting isolated NSs have been made in $90 \mathrm{~s}$ (see a review in Treves et al. (2000) PASP 112, 297).

Since then researches became a little bit pessimistic about the subject. However, with present day abilities and prospects for near future

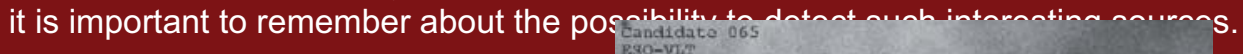

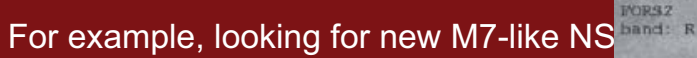
which are expected to be more abundan optimistic scenario) at fluxes $<10^{-13} \mathrm{erg} / \mathrm{c}$

Recently, Pires and Motch (0710.5192) in the 2XMMp catalogue. One interestin Most probably, it is a cooling INS (work

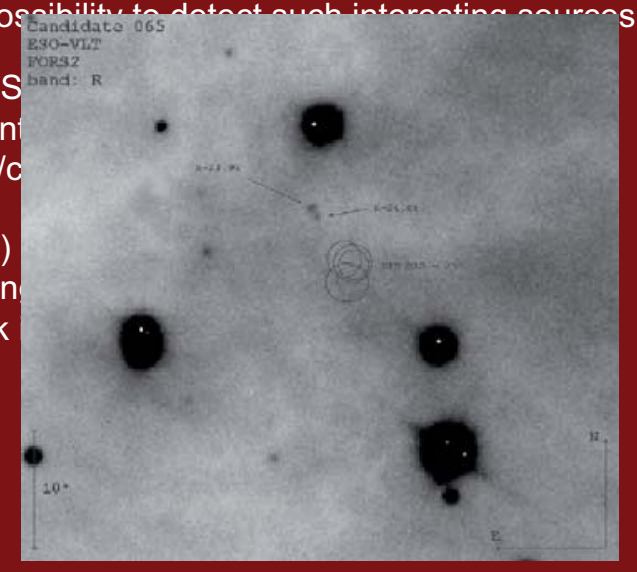

\section{Looking for radio pulsar counterparts for EGRET unidentified sources}

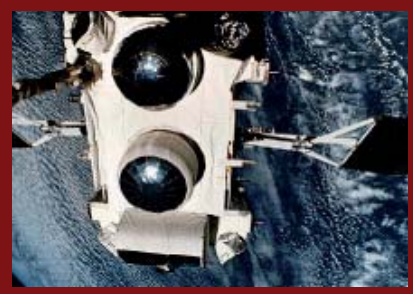

Recently Crawford et al. (astro-ph/0608225) tried to find dim radio pulsars in 56 relatively small error boxes of EGRET unidentified sources. Nothing came out.

Then, Keith et al. (0807.2088) made a search at high frequencies for three cases and discovered a new pulsar! Probably, it is important to use high frequencies ( few GHz)

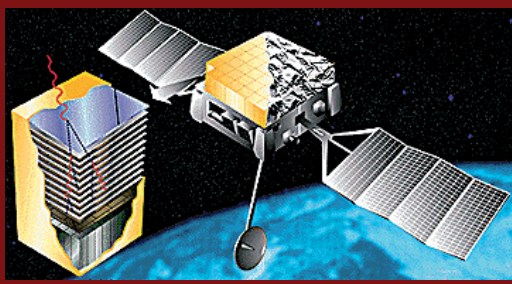

GLAST is in orbit now and everything is working. Hopefully, soon we'll have more gamma-ray selected isolated neutron stars (radio pulsars, coolers, ....).

More population studies will be necessary which take into account all possible types of NSs. 


\section{Conclusions}

- Population synthesis is a useful tool in astrophysics

- Many theoretical parameters can be tested only via such modeling

- Many parameters can be determined only via PS models

- Actively used to study NSs

- Actively used for predicting future observations and setting on observational programs

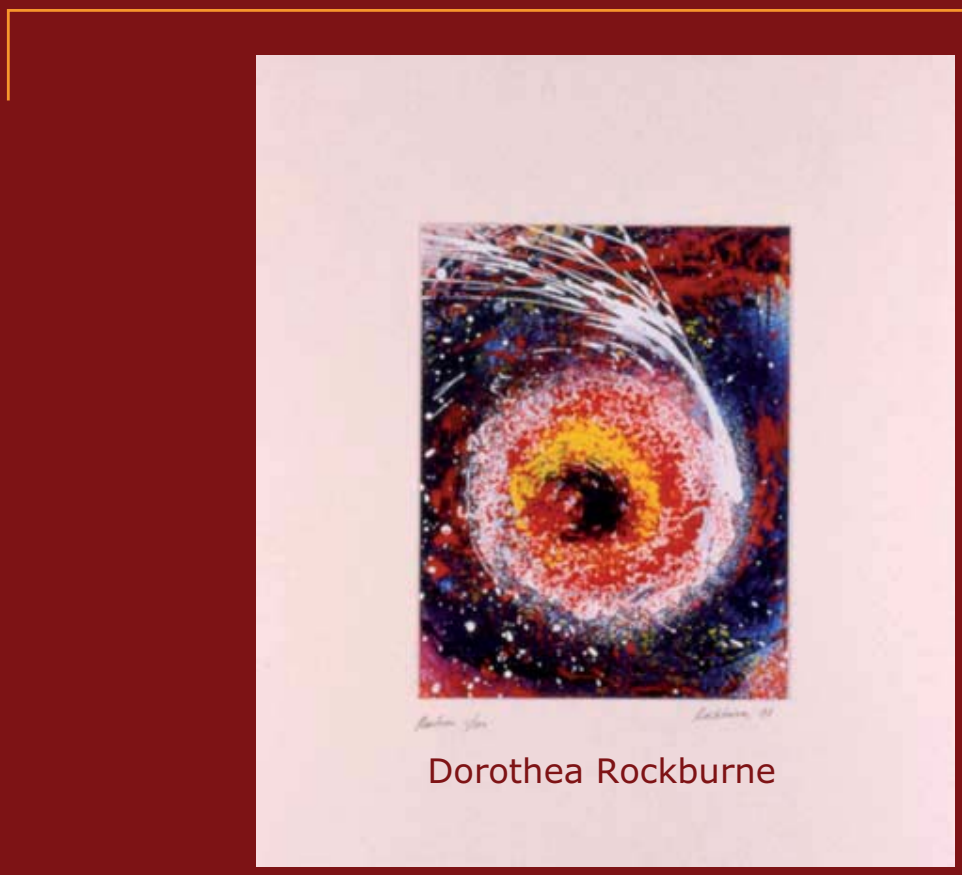




\section{Papers to read}

- Popov, Prokhorov "Population synthesis in Astrophysics"

Physics-Uspekhi 50 (11), 1123 (2007)

- Faucher-Giguere, Kaspi "Birth and evolution of isolated radio pulsars" astro-ph/0512585

- Postnov, Yungelson "The Evolution of Compact Binary Star Systems "

Living Reviews on Relativity 9, 6 (2006) astro-ph/0701059

- Lipunov et al. "Description of the Scenario Machine" arXiv: 0704/1387

- Lipunov, Postnov, Prokhorov "The Scenario Machine:

Binary Star Population Synthesis"

Astrophysics and Space Science Reviews (1996)

http://xray.sai.msu.ru/ mystery/articles/review/ 